ACCEPted to ApJ

Preprint typeset using $\mathrm{IAT}_{\mathrm{E}} \mathrm{X}$ style emulateapj v. 5/2/11

\title{
SPATIALLY RESOLVED CHEMISTRY IN NEARBY GALAXIES II. THE NUCLEAR BAR IN MAFFEI 2
}

\author{
DAVID S. MEIER ${ }^{1,2}$, AND JEAN L. TURneR ${ }^{3}$ \\ (Received)
}

Accepted to ApJ

\begin{abstract}
We present $2^{\prime \prime}-10^{\prime \prime}$ imaging of eleven transitions from nine molecular species across the nuclear bar in Maffei 2. The data were obtained with the BIMA and OVRO interferometers. The ten detected transitions are compared with existing CO isotopologues, HCN, CS and millimeter continuum data. Dramatic spatial variations among the mapped species are observed across the nuclear bar. A principle component analysis is performed to characterize correlations between the transitions, star formation and molecular column density. The analysis reveals that $\mathrm{HCN}, \mathrm{HNC}, \mathrm{HCO}^{+}$and 3 $\mathrm{mm}$ continuum are tightly correlated, indicating a direct connection to massive star formation. We find two main morphologically distinct chemical groups, $\mathrm{CH}_{3} \mathrm{OH}, \mathrm{SiO}$ and $\mathrm{HNCO}$ comprising the grain chemistry molecules, versus $\mathrm{HCN}, \mathrm{HNC}, \mathrm{HCO}^{+}$and $\mathrm{C}_{2} \mathrm{H}$, molecules strong in the presence of star formation. The grain chemistry molecules, $\mathrm{HNCO}, \mathrm{CH}_{3} \mathrm{OH}$ and $\mathrm{SiO}$, trace hydrodynamical bar shocks. The near constancy of the $\mathrm{HNCO} / \mathrm{CH}_{3} \mathrm{OH}, \mathrm{SiO} / \mathrm{CH}_{3} \mathrm{OH}$ and $\mathrm{SiO} / \mathrm{HNCO}$ ratios argue that shock properties are uniform across the nucleus. $\mathrm{HCN} / \mathrm{HCO}^{+}, \mathrm{HCN} / \mathrm{HNC}, \mathrm{HCN} / \mathrm{CS}$ and $\mathrm{HCN} / \mathrm{CO}$ ratios are explained primarily by variations in density. High $\mathrm{HCO}^{+} / \mathrm{N}_{2} \mathrm{H}^{+}$ratios are correlated with the $\mathrm{C}_{2} \mathrm{H}$ line, suggesting that this ratio may be a powerful new dense photon-dominated region (PDR) probe in external galaxies. $\mathrm{C}_{2} \mathrm{H}$ reveals a molecular outflow along the minor axis. The morphology and kinematics of the outflow are consistent with an outflow age of 6-7 Myrs.
\end{abstract}

Subject headings: galaxies: individual(Maffei 2) — galaxies: starburst — galaxies: ISM — radio lines: galaxies - astrochemistry

\section{INTRODUCTION}

Molecular abundances and excitation are sensitive probes of physical processes taking place in molecular clouds (e.g., van Dishoeck \& Blake 1998). Chemistry is pivotal both in controlling gas cooling and ionization balance as well as in revealing changing cloud physical conditions resulting from shocks and feedback from massive star formation and galactic structure. Millimeter telescopes are now capable of surveying many molecular transitions in other galaxies, opening up chemical studies to the rest of the Universe.

Single dish millimeter line surveys show that spectra of external galaxies are extremely rich in molecular lines (e.g. Usero et al. 2004; Martín et al. 2006; Costagliola et al. 2011; Snell et al. 2011; Aladro et al. 2011b). For nearby galaxies, giant molecular cloud (GMC) scale chemistry is within reach of current millimeter interferometers. In the first paper of this series (Meier \& Turner 2005; hereafter MT05), we exploited this richness to carry out the first chemical imaging survey of the nucleus of an external galaxy with spatial resolution high enough to separate individual GMCs. Images of eight astrochemically important species were obtained in the nearby spiral IC 342, revealing strong chemical differentiation among the GMCs. A principal component analysis demonstrated that the chemical variation is strongly correlated with galactic structure such as bar and arm features, as well as the presence and evolu-

\footnotetext{
${ }^{1}$ New Mexico Institute of Mining \& Technology, 801 Leroy Place, Socorro, NM 87801; dmeier@nmt.edu

2 National Radio Astronomy Observatory, P. O. Box O, 1003 Lopezville Road, Socorro, NM 87801

${ }^{3}$ Department of Physics and Astronomy, UCLA, Los Angeles, CA 90095-1562; turner@astro.ucla.edu
}

tionary state of star forming regions (MT05; Meier et al. 2011).

In this paper we extend the analysis to the nucleus of a second nearby spiral galaxy, Maffei 2. We have two main goals for this survey. The first is to test the generalizability of the results discovered in MT05. Maffei 2 and IC 342 are at the same distance and their nuclear morphologies are similar. Both have two molecular spiral arms terminating on a central ring, with the central ring being the site of intense, young, massive star formation. It is reasonable to expect that the two nuclei should exhibit similar chemistries if the results of MT05 for IC 342 are applicable to barred spiral nuclei in general.

The second goal is to investigate the chemistry of Maffei 2 with particular attention focused on its unique features. MT05 find that in IC 342, shocks appear to be an important influence on the nuclear chemistry and speculate that the shocks are associated with orbital resonances in a bar potential. The bar in IC 342 is weak (Crosthwaite et al. 2001); however, and there remain other explanations for the nuclear morphology (Schinnerer et al. 2008). It is important to follow up with an object that has a well established, strong bar. Kinematic modeling leaves little doubt that the nucleus of Maffei 2 hosts a strong, highly defined nuclear bar of 320 pc radius (Meier et al. 2008, hereafter MTH08; Kuno et al. 2008). Because of the strength of the bar, we expect that shock chemistry should be more important than in IC 342. Maffei 2 also has a somewhat more intense and extended starburst than IC 342 (Turner \& Ho 1983, 1994), so photon-dominated region (PDR) effects may be more prominent than they are in IC 342 .

\section{OBSERVATIONS}


To pursue these goals, we obtain aperture synthesis observations of eleven important molecular transitions over the central $\mathrm{R} \sim 0.5 \mathrm{kpc}$ of the nuclear bar in Maffei 2, for comparison with $\mathrm{CO}$ isotopologues (Hurt \& Turner 1991; MTH08), HCN(1-0) (MTH08) and CS (Kuno et al. 2008). Table 1 lists molecular parameters for each transition. With the Owens Valley Radio Observatory (OVRO) millimeter interferometer we observed three lines, $\mathrm{HCO}^{+}(1-0), \mathrm{HNCO}\left(5_{05^{-}} 4_{04}\right)$ and $\mathrm{SiO}(1-0 ; v=1)$. $\mathrm{HCO}^{+}(1-0)$ and $\mathrm{SiO}(1-0 ; v=$ 1) were observed between 1999 January 28 and 1999 March 29 along with the $\mathrm{HCN}(1-0)$ dataset (MTH08). $\operatorname{HNCO}\left(5_{05}-4_{04}\right)$ was observed along with ${ }^{13} \mathrm{CO}(1-0)$ and $\mathrm{C}^{18} \mathrm{O}(1-0)$ between 1998 October 19 and 1999 January 5 (Table 1). The OVRO interferometer consisted of six 10.4 meter antennas equipped with SIS receivers (Padin et al. 1991; Scoville et al. 1994). Transitions were observed in L, $\mathrm{H}$ and UH array configurations except for the $\mathrm{HNCO}$, which was observed in $\mathrm{L}$ and $\mathrm{H}$, providing spatial resolutions of $\sim 2$." 5 (Table 1). Data were calibrated using the in-house OVRO calibration package, MMA. Phase calibration was done by observing the point source $0224+671$ every 20 minutes. Absolute flux calibration was based on observations of Neptune or Uranus with 3C 273, 3C 84 and 3C 454.3 as supplementary flux calibrators. Based on the derived fluxes and flux histories of these secondary flux calibrators we estimate that the absolute fluxes are good to $10-15 \%$. Two pointings were mosaiced to cover the nuclear bar and therefore maps are corrected from the primary beam response.

The eight remaining transitions were observed with the ten element Berkeley - Illinois - Maryland Association (BIMA) 4 array (Welch et al. 1996) between 2002 September 08 and 2003 March 18 (Table 1). The eight spectral lines were observed in two sets of spectrometer configurations. $\mathrm{C}_{2} \mathrm{H}\left(\mathrm{N}=1-0, \mathrm{~J}=\frac{3}{2}-\frac{1}{2}\right), \mathrm{C}_{2} \mathrm{H}(\mathrm{N}=1-$ $\left.0, \mathrm{~J}=\frac{1}{2}-\frac{1}{2}\right), \mathrm{SiO}(2-1 ; v=0), \mathrm{HNC}(1-0)$ and $\mathrm{HC}_{3} \mathrm{~N}(10$ 9) were observed together, as were $\mathrm{CH}_{3} \mathrm{OH}\left(2_{k}-1_{k}\right)$, $\mathrm{C}^{34} \mathrm{~S}(2-1)$ and $\mathrm{N}_{2} \mathrm{H}^{+}(1-0)$. Data were obtained in $\mathrm{B}, \mathrm{C}$ and $\mathrm{D}$ configurations for all transitions giving beamsizes of $\sim 7^{\prime \prime}-10^{\prime \prime}$ depending on frequency and sensitivity. Calibration and data reduction was done in MIRIAD. Phase calibration was also done by observing the same point source $0224+671(0228+673)$ interlaced with the source every 24 minutes. Absolute flux calibration was done by observing $\mathrm{W} 3(\mathrm{OH})$. Based on the derived fluxes and flux histories of $0224+671$ we estimate that the uncertainties in absolute flux are $10-15 \%$, similar to the OVRO data. The $\sim 130^{\prime \prime}$ FWHM primary beam of the BIMA antenna is large enough such that only one pointing was required. No corrections for primary beam attenuation have been applied.

Image analysis was done with the NRAO AIPS package. In making the integrated intensity maps emission greater than $1.2 \sigma$ was included. Continuum emission has not been subtracted from the maps since the $3 \mathrm{~mm}$ continuum peak is below $1 \sigma$.

\section{RESULTS}

\footnotetext{
${ }^{4}$ Operated by the University of California, Berkeley, the University of Illinois, and the University of Maryland with support from the National Science Foundation.
}

\subsection{A Sketch of the Nuclear Region of Maffei 2}

The Sb galaxy Maffei 2 is one of the nearest and brightest extragalactic molecular line sources, at a distance of only 3.3 Mpc $\left(1^{\prime \prime} \sim 15 \mathrm{pc}\right.$, Fingerhut et al. 2007, see discussion in MTH08). A strongly barred and somewhat asymmetric galaxy, Maffei 2 has undergone a recent interaction with a small companion that has driven a large quantity of gas into the nucleus, building up a compact central bulge seen in the NIR (Hurt et al. 1993, 1996). This bar-like compact bulge, has further driven the molecular gas into a central molecular zone of radius $R \sim 300 \mathrm{pc}$ that is barred and highly inclined (Ishiguro et al. 1989; Hurt \& Turner 1991). This nuclear bar, containing $\mathrm{M}\left(\mathrm{H}_{2}\right) \sim 2 \times 10^{7} \mathrm{M}_{\odot}$ of gas (MTH08), is structurally reminiscent of a miniature version of large scale gaseous bars (Athanassoula 1992) (Fig. 11). Using $2 \mathrm{~cm}$ and $3 \mathrm{~mm}$ radio / $\mathrm{mm}$ continuum to trace ionizing radiation it is seen that the intersections of bar arms and central ring are sites of intense localized star formation $\left(\mathrm{SFR} \sim 0.04 \mathrm{M}_{\odot} \mathrm{yr}^{-1}\right.$ per $\mathrm{GMC}$ or a total of $\mathrm{L}_{\mathrm{OB}} \sim 10^{8} \mathrm{~L}_{\odot}$; Turner \& Ho 1994; Tsai et al. 2006; MTH08). Averaged over the inner $\sim 20^{\prime \prime}$ radius, gas consumption timescales decrease below 100 Myrs, making Maffei 2's nucleus a starburst.

A central molecular ring of radius $\sim 7^{\prime \prime}(110 \mathrm{pc})$ is clearly resolved in gas kinematics, although the ring is less apparent in integrated intensity images (MTH08). GMCs D, E, and F (cloud designations following MTH08 are shown in Figs. 1) are located along the eastern side of the nuclear ring. Bar arms (including GMCs B and $\mathrm{C}$ in the north and $\mathrm{G}$ and $\mathrm{H}$ in the south) extend off the leading edges of the ring. The bright GMCs of the central ring, typically $\sim 60-80 \mathrm{pc}$ in extent, and with masses of a few $10^{6} \mathrm{M}_{\odot}$, delineate the sites where gas flowing in along the bar arms piles up at the inner ring. Dense gas traced by HCN preferentially picks out the arm-ring intersections. Gas not incorporated into the dense component at the starburst locations is tidally sheared into a moderate density, smoothly distributed ring (MTH08). Massive star formation is largely absent in the western side of the ring (MTH08). Based on $\mathrm{HCN}$, the fraction of dense gas is significantly lower towards the bar ends. Densities derived from $\mathrm{CO}$ tend to be fairly constant $\left(\mathrm{n}_{H_{2}} \sim 10^{3}\right)$ over the inner bar, with the clouds associated with young star formation slightly warmer $\left(T_{K} \sim 30-40 \mathrm{~K}\right)$ than the others $\left(\mathrm{T}_{k} \sim 20 \mathrm{~K}\right)$. Gas clouds on the quiescent western side of the nuclear ring are slightly cooler yet (MTH08).

\subsection{Molecular Abundances in Maffei 2}

Column densities are determined assuming optically thin emission, and LTE:

$$
N_{m o l}=\left(\frac{3 k Q e^{E_{u} / k T_{e x}}}{8 \pi^{3} \nu S_{u l} \mu_{0}^{2} g_{K_{u}} g_{I_{u}}}\right) I_{m o l},
$$

where $S_{u l}, g$ and $E_{u}$ are the line strength, degeneracy and upper energy of each state, respectively, and $\mathrm{T}_{\mathrm{ex}}$ is the excitation temperature associated with the transition. Given that we have mapped only one transition of each species, corrections for background radiation and opacity have been ignored. Column densities are sensitive to $\mathrm{T}_{\mathrm{ex}}$ through the partition function, $Q$, and the 
energy of the upper state. The asymmetric tops (HNCO and $\mathrm{CH}_{3} \mathrm{OH}$ ) are more sensitive to temperature changes than are the linear rotors. Changes in gas density also affect excitation, particularly for molecules with high critical densities ( $\mathrm{HNC}, \mathrm{N}_{2} \mathrm{H}^{+}$and $\mathrm{HC}_{3} \mathrm{~N}$ ). Densities have been at least partially constrained by observation in Maffei 2 (MTH08). We adopt a $\mathrm{T}_{e x}=10 \mathrm{~K}$ for the molecular transitions here. This is similar to what is derived from the ${ }^{13} \mathrm{CO}$ data (MTH08) and is the same as assumed for IC 342 (MT05), permitting easy comparison. However $\mathrm{T}_{e x}=10 \mathrm{~K}$ may slightly underestimate excitation at the massive star forming regions. Measured line intensities, peak temperatures, line centroids and line widths for the GMCs are reported in Table 1. Fractional abundances $\left(X(\mathrm{~mol}) \equiv \mathrm{N}_{\mathrm{mol}} / \mathrm{N}_{\mathrm{H}_{2}}\right)$ calculated from the intensities given the above assumptions are listed in Table 1 based on molecular parameters in Table 1.

Fractional abundances also require an $\mathrm{H}_{2}$ column density, $\mathrm{N}\left(\mathrm{H}_{2}\right)$. $\mathrm{N}\left(\mathrm{H}_{2}\right)$ is most easily obtained from the $\mathrm{CO}(1-0)$ brightness and an empirical Galactic conversion factor, $\mathrm{X}_{\mathrm{CO}}$. However, $\mathrm{X}_{\mathrm{CO}}$ overpredicts $\mathrm{N}\left(\mathrm{H}_{2}\right)$ in nearby galaxy centers, including Maffei 2 , by factors of a few (MTH08). An alternative measure of $\mathrm{N}\left(\mathrm{H}_{2}\right)$ can be obtained from the $\mathrm{CO}$ isotopologues, which tend to be optically thin. We use the ${ }^{13} \mathrm{CO}(1-0)$ integrated intensity and $\left[\mathrm{H}_{2} /{ }^{13} \mathrm{CO}\right]=7.0 \times 10^{5}\left(\left[{ }^{12} \mathrm{CO} /{ }^{13} \mathrm{O}\right]=60\right.$ and $\left[\mathrm{CO} / \mathrm{H}_{2}\right]=8.5 \times 10^{-5}$ ) when calculating the $\mathrm{H}_{2}$ column densities (see MTH08, for a detailed discussion of $\mathrm{N}\left(\mathrm{H}_{2}\right)$ and its uncertainties in Maffei 2).

Fractional abundances for the species below are estimated to be uncertain to at least a factor of three, although the relative column densities - that is, the relative spatial distributions within the nucleus of the different molecules - should be more reliable. MT05 has a detailed discussion of the uncertainties associated with the abundance estimates. Table 1 records for reference, the change in derived column densities if $\mathrm{T}_{e x}$ is changed from $10 \mathrm{~K}$ to $50 \mathrm{~K}$, more typical of the large beam gas kinetic temperatures.

\subsection{The Molecules of Maffei 2}

We now discuss the morphologies of each species. Spectra taken at selected locations across the nucleus with $8^{\prime \prime}$ apertures are shown for each transition in Fig. 2 Fig. 3 displays maps of the transitions detected with OVRO and Fig. 4 displays those detected with BIMA. In the subsequent analysis we also include $3 \mathrm{~mm}$ continuum emission and CO isotopologues from MTH08. The millimeter continuum in Maffei 2 is dominated by freefree emission rather than dust emission (MTH08).

SiO - Silicon Monoxide: The $\mathrm{J}=2-1$ rotational line of both the $v=0$ and 1 vibrational states were observed. The $v=1, \mathrm{~J}=2-1$ transition is a maser in the Galaxy (Snyder \& Buhl 1975), but we do not detect it in Maffei 2. The thermal $v=0$ transition of $\mathrm{SiO}$ is tentatively detected toward GMCs B, F and $\mathrm{G}$ with peak temperatures over the $8^{\prime \prime}$ aperture of 35 $\mathrm{mK}$ (Fig. 4). The integrated intensity map is noisy but consistent in morphology to $\mathrm{HNCO}$ and $\mathrm{CH}_{3} \mathrm{OH}$, extended along the southern arm. Implied $\mathrm{SiO}$ abundances reach $\sim 1 \times 10^{-10}$. This abundance is lower than estimated for IC 342 and the nearby starburst nuclei, NGC 253 and M 82 (García-Burillo et al. 2000, 2001;
Usero et al. 2006), but typical of diffuse Galactic clouds (Greaves et al. 1996; Turner 1998b) away from molecular outflows (Martín-Pintado et al. 1992); it is much larger than what is found for Galactic dark clouds (e.g., Ziurvs et al. 1989).

$\mathrm{C}_{2} \mathrm{H}-$ Ethynyl: We detect both the $\mathrm{J}=\frac{3}{2}-\frac{1}{2}$ and $\frac{1}{2}-\frac{1}{2}$ fine structure components of the $\mathrm{N}=1-0$ line. $\mathrm{C}_{2}^{2} \mathrm{H}$ is brightest towards the northern star forming ring (GMC $\mathrm{D}+\mathrm{E})$, peaking at $0.11 \mathrm{~K}$, and weakens significantly along the bar arms (Fig. 4). Towards GMCs F and $\mathrm{G}, \mathrm{C}_{2} \mathrm{H}$ avoids the column density peaks traced in ${ }^{13} \mathrm{CO}(1-0)$. Line widths are significantly broader than those seen in the other weaker lines, so the hyperfine structure of each fine structure component is being marginally resolved here. Evidence for the most blueshifted $\mathrm{N}=1-0, \mathrm{~J}$ $=\frac{1}{2}-\frac{1}{2}, \mathrm{~F}=1-0$ hyperfine component is seen towards the brightest GMCs (see Fig. 2). There is a plume of emission along the minor axis in the $\frac{3}{2}-\frac{1}{2}$ line. From the relative intensities of the fine structure lines we can determine the $\mathrm{C}_{2} \mathrm{H}$ opacity. The ratio of the brightness temperature of the two fine structure transitions, $\mathrm{I}\left(\frac{3}{2}-\frac{1}{2}\right) / \mathrm{I}\left(\frac{1}{2}-\frac{1}{2}\right)$ ranges from $1.2-\sim 2.8$, with lowest values toward the regions with the brighter emission. For optically thin, LTE excitation a this ratio should be 2.0. Therefore $\mathrm{C}_{2} \mathrm{H}$ opacities are significant along the central ring, with implied values for the $\frac{3}{2}-\frac{1}{2}$ transition of $\tau_{C C H} \simeq 1.5$. Along the arms $\tau_{C C H}<1$ except at regions around $\mathrm{GMC} \mathrm{G}$ away from the ${ }^{13} \mathrm{CO}(1-0)$ column density peaks where $\tau_{C C H} \sim 3$. Hence, despite its lower signal-to-noise, the $\frac{1}{2}-\frac{1}{2}$ transition probably represents a closer picture of the true $\mathrm{C}_{2} \mathrm{H}$ abundance distribution. Fractional abundances assuming $\mathrm{C}_{2} \mathrm{H}$ is optically thin peak at $\sim 2.5 \times 10^{-8}$. If we account for opacity, the abundance rises to $\sim 4.8 \times 10^{-8}$. These are similar to those seen towards the PDR site in IC 342 (MT05) and at the high end of the range seen in Galactic cores (Wootten et al. 1980; Huggins et al. 1984; Watt 1983; Turner et al. 1999; Lucas \& Liszt 2000).

$\mathrm{HCO}^{+}-$Formyl Ion: $\mathrm{HCO}^{+}(1-0)$ is the brightest line of this sample, and is imaged at high resolution $\left(\sim 2^{\prime \prime}\right)$. The emission is dominated by GMCs $\mathrm{D}+\mathrm{E}$ and $\mathrm{F}$, with line peaks of $2.0 \mathrm{~K}\left(0.58 \mathrm{~K}\right.$ over the $8^{\prime \prime}$ aperture) (Fig. 3). Like $\mathrm{HCN}(1-0)$ (MTH08), $\mathrm{HCO}^{+}(1-0)$ is primarily confined to the central ring region. There is a second blueshifted velocity component at $\sim-135 \mathrm{~km} \mathrm{~s}^{-1}$ (LSR) along the northern portion of the bar. $\mathrm{HCO}^{+}(1-0)$ abundances in Maffei 2 are between $2-8 \times 10^{-9}$, but these (along with those of HCN and HNC) should be considered lower limits since these very bright transitions may have significant optical depths (see section 4.1.1). These agree well with the $\mathrm{HCO}^{+}$abundances in most Galactic dense clouds (eg. Pirogov et al. 1995; Lucas \& Liszt 1996; Turner 1995a) and single-dish extragalactic measurements (Nguven-Q-Rieu et al. 1992).

$H N C-H y d r o g e n$ Isocyanide: The $\mathrm{J}=1-0$ line of the linear molecule HNC is bright over the whole nuclear bar, with peak temperatures $\sim 0.25 \mathrm{~K}$ (Fig. 4). At this resolution, the morphology matches closely both ${ }^{13} \mathrm{CO}(1-0)$ and $\mathrm{HCN}(1-0)$, being dominated by the bright clouds, D $+\mathrm{E}$ and $\mathrm{F}$, but HNC emission is seen from every cloud including the non-nuclear bar GMC, A. Towards the northern side of the bar HNC emission is especially bright at 
higher blueshifted velocities, similar to the component seen in $\mathrm{HCO}^{+}(1-0)$. HNC abundances are $1-2 \times 10^{-9}$, in agreement with IC 342 (MT05). These abundances are intermediate between typical massive star forming cores and Galactic dark clouds (Wootten et al. 1978; Blake et al. 1987; Hirota et al. 1998; Nummelin et al. 2000).

$\mathrm{HC}_{3} \mathrm{~N}$-Cyanoacetylene: We mapped the $\mathrm{J}=10-9$ rotational line of $\mathrm{HC}_{3} \mathrm{~N}$. This molecule has the largest electric dipole moment and the highest upper energy state of the (thermal) sample (Table11). It is thus not surprising that we observe $\mathrm{HC}_{3} \mathrm{~N}$ to be faint and spatially confined (Fig. 4). To improve $\mathrm{S} / \mathrm{N}$, the $\mathrm{HC}_{3} \mathrm{~N}$ map was smoothed to a slightly larger beam size, but even then peaks at only $\sim$ $50 \mathrm{mK}$ towards two unresolved locations at the intersection of the ring and bar arms $(\mathrm{C}$ and $\mathrm{F})$. Abundances are $\sim 10^{-9}$, similar to those in IC 342 (MT05; Meier et al. 2011), somewhat higher than in cold Galactic clouds but much lower than the localized Galactic hot core values (>10 ${ }^{-7}$; eg. Morris et al. 1976; Vanden Bout et al. 1983; Chung et al. 1991; de Vicente et al. 2000).

$\mathrm{N}_{2} \mathrm{H}^{+}$-Diazenylium: In the Galaxy, $\mathrm{N}_{2} \mathrm{H}^{+}$is regarded as a tracer of quiescent gas. We observed the $\mathrm{J}=1-0$ rotational state of this linear ion, which has hyperfine splitting much smaller than the observed linewidths. As is the case in $\mathrm{IC} 342, \mathrm{~N}_{2} \mathrm{H}^{+}$is bright and extended across the nucleus (Fig. 4). Emission peaks towards GMC F with a peak brightness temperature reaching $\sim 0.15 \mathrm{~K}$. GMCs $\mathrm{D}$ and $\mathrm{E}$, the closest clouds to the northern star formation region, show weak emission in $\mathrm{N}_{2} \mathrm{H}^{+}: X\left(\mathrm{~N}_{2} \mathrm{H}^{+}\right)$ reaches $\sim 7 \times 10^{-10}$ at GMC $\mathrm{F}$ and drops by nearly a factor of three towards the $3 \mathrm{~mm}$ continuum peak. The highest abundances are similar to what is seen in IC 342 and dense, quiescent cores in the Galactic disk (Womack et al. 1992; Benson et al. 1998).

$C^{34} S$-Carbon Monosulfide: This is an isotopologue of the dense gas tracer $\mathrm{CS}$. The $\mathrm{J}=2-1$ rotational transition of $\mathrm{C}^{34} \mathrm{~S}$ is tentatively detected only towards GMCs A, C and F, at $<30 \mathrm{mK}$ (Fig. 4). The tentative detections imply $X\left(\mathrm{C}^{34} \mathrm{~S}\right)$ abundances of at most a few $\times 10^{-10}$, typical of Galactic dense cores and diffuse/translucent clouds (for a $\mathrm{C}^{32} \mathrm{~S} / \mathrm{C}^{34} \mathrm{~S}$ isotopic abundance of 23; eg. Nyman 1984; Drdla et al. 1989; Wang et al. 1993; Chin et al. 1996; Morata et al. 1997; Lapinov et al. 1998; Lucas \& Liszt 2002). In the GMCs near the northern star forming region abundances are an order of magnitude lower. Evidently $\mathrm{C}^{34} \mathrm{~S}(2-1)$ is not strongly enhanced anywhere across the nucleus, and especially not at the massive star formation sites. Limits on the $\mathrm{CS} / \mathrm{C}^{34} \mathrm{~S}$ isotopologue ratio assuming optically thin CS ranges from 4 to $>14$ based on the $\mathrm{CS}(2-1)$ line of Kuno et al. (2008). The tentative detections of $\mathrm{CS} / \mathrm{C}^{34} \mathrm{~S}$ $\sim 4$ suggests opacities significantly greater than unity in $\mathrm{CS}(2-1)$.

$\mathrm{CH}_{3} \mathrm{OH}-$ Methanol: We observed the low energy, blended set of $2_{1}-1_{1} \mathrm{E}, 2_{0}-1_{0} \mathrm{E}, 2_{0}-1_{0} \mathrm{~A}+$ and $2_{-1}-1_{-1} \mathrm{E}$ thermal transitions of $\mathrm{CH}_{3} \mathrm{OH}$ (hereafter labeled the $2_{k}-1_{k}$ transition). We cannot resolve these transitions in this group, given the source linewidths. The $2_{k}-1_{k}$ transition of $\mathrm{CH}_{3} \mathrm{OH}$ is very bright across the nucleus, with peak temperatures reaching $0.28 \mathrm{~K}$ (Fig. 4). Of the species observed in this paper only $\mathrm{HCO}^{+}(1-0)$ is brighter. Remarkably though, the mor- phology of $\mathrm{CH}_{3} \mathrm{OH}$ is completely different from what is seen in most of the other lines, including ${ }^{13} \mathrm{CO}(1-0)$ and $\mathrm{HCN}(1-0) . \mathrm{CH}_{3} \mathrm{OH}$ peaks strongly towards the southern nuclear bar end $(\mathrm{G}$ and $\mathrm{H})$ and the southern nuclear bar arm / ring intersection region $(\mathrm{F})$. By contrast, emission is barely detected near GMC D+E. The emission favors the right side of the southern arm, which is the trailing, upstream side. At the bar ends methanol abundances peak at $\sim 1.5 \times 10^{-8}$. Towards GMC D abundances drop by a factor of 5 . The peak arm abundances are a factor of two higher than those of IC 342 (MT05). Galactic methanol abundances can reach these levels in small localized hot cores regions (eg. Kalenskii \& Sobolev 1994; Kalenskii et al. 1997; Bachiller \& Perez Gutierrez 1997; Turner 1998a; Nummelin et al. 2000; Minier \& Booth 2002). $\mathrm{CH}_{3} \mathrm{OH}$ velocities appear consistently blueshifted with respect to most of the other transitions. In the north the velocity shift is $\sim-10 \mathrm{~km} \mathrm{~s}^{-1}$, growing to $\sim$ $-40 \mathrm{~km} \mathrm{~s}^{-1}$ along the southern bar end. The shift towards the northern cloud is not significant, however the shift seen towards GMCs F and G appears real. This may result from an increased population in the higher excitation blueshifted lines that make up the quadruplet or somewhat different kinematics due to the presence of shocks (see section 4.4.1).

HNCO -Isocyanic Acid: We observed the $\mathrm{K}_{-1}=0$ transition of the $J=5-4$ rotational state $\left(5_{05}-4_{04}\right)$ of this prolate, slightly asymmetric top. In the higher resolution OVRO maps, HNCO peaks at GMCs F and G, with faint emission along the bar connecting the two clouds (Fig. 3). When smoothed to match the resolution of the BIMA data, $\mathrm{HNCO}$ has a very similar morphology to $\mathrm{CH}_{3} \mathrm{OH}$. Over the $8^{\prime \prime}$ aperture used for the spectra in Fig. 2, HNCO has a peak brightness of $0.25 \mathrm{~K}$, whereas over the $40 \mathrm{pc}$ resolution achieved in the OVRO data peak brightnesses reach $1.0 \mathrm{~K}$. No emission is detected towards GMC $\mathrm{D}$ in the high resolution map. HNCO abundances reach $X(\mathrm{HNCO}) \simeq 9 \times 10^{-9}$ along the southern nuclear bar. These abundances are typical of those observed on one to two orders of magnitude smaller scales in Galactic massive dense cores (Zinchenko et al. 2000) and are even a factor of 3 larger than the $\mathrm{HNCO}$ enhanced shock regions in IC 342 (MT05). $\mathrm{CH}_{3} \mathrm{OH}$ and HNCO in Maffei 2 have some of the most pronounced morphological differences from $\mathrm{CO}$ yet seen in extragalactic sources. These two species illustrate the importance of high spatial resolution interferometric observations of chemical species.

Other Transitions: Table 1 presents the limits $(2 \sigma)$ for other potentially detectable transitions within the observed bandwidth. These include the ${ }^{13} \mathrm{C}$ substituted isotopologues of $\mathrm{HNC}(1-0)$ and $\mathrm{HC}_{3} \mathrm{~N}(10-9)$ and two transitions of the radical $\mathrm{HCO}$. None of these transitions are detected. In the case of the ${ }^{13} \mathrm{C}$ isotopologues only the $\mathrm{HN}^{13} \mathrm{C}(1-0)$ limits are at all constraining (Table 1). Towards the central ring and southern bar end $1 \sigma \mathrm{HNC}(1-$ $0) / \mathrm{HN}^{13} \mathrm{C}(1-0)$ line ratio lower limits are $>10-16$, which in these locations are consistent with or slightly above the corresponding $\mathrm{CO}$ isotopic line ratios (MTH08). Therefore the $\mathrm{HNC}(1-0)$ opacities are lower than ${ }^{12} \mathrm{CO}(1-0)$ opacities.

To facilitate the exposition, we omit the transitions for each species where possible in the remainder of the text. We discuss transition dependent effects specifically 
where important.

\subsection{Understanding the Molecular Maps: Principal Component Analysis}

The maps give a picture of the molecular cloud astrochemistry in the nuclear region of Maffei 2. To interpret these maps, we need to establish similarities and differences between the molecules. The correlations reveal trends in the chemistry, which in turn, can suggest the physical driving forces responsible for these characteristics and how they change across the nucleus.

As in MT05, we quantify morphological correlations amongst the images via a principal component analysis (PCA). PCA is a common technique (eg. Murtage \& Heck 1987; Krzanowski 1988; Everitt \& Dunn 2001; Wall \& Jenkins 2003) used to reduce the dimensionality of a dataset. The PCA simplifies the picture of molecular distribution, reducing a large amount of information to a few images, providing an excellent framework within which to study the complex variations in molecular properties in Maffei 2. For a general description of PCA applied to multi-transition molecular maps, see Hever \& Schloerb (1997) or for specific applications to extragalactic molecular maps, see MT05. The PCA is dependent on the particular set of data given to it; here, we are analyzing the correlations of $3 \mathrm{~mm}$ lines of heavy molecules, so the results we obtain pertain to the cool and relatively dense cloud population in Maffei 2.

To calculate the principal components for Maffei 2, the line maps were remade to the same geometry and beamsize (that of HNC; Table 11), normalized and meancentered. Thirteen maps, including ${ }^{13} \mathrm{CO}(1-0), \mathrm{HCN}(1-$ $0)$ and $3 \mathrm{~mm}$ continuum from MTH08, plus the ten transitions of the nine species discussed in section 3.3 were sampled at $1^{\prime \prime}$ intervals over the central $38^{\prime \prime} \times 56^{\prime \prime}$, making up a 27664 element data-space. The algorithm used to calculate the principal components is that of Murtage \& Heck (1987).

The results of the PCA are tabulated in Tables 1 and 1. Table 1 shows the matrix of pair-wise correlation coefficients between all of the thirteen maps. Table1 1 records the projection of each map onto the seven modeled PCs. Fig. 5 displays the maps of the first three (most important) principal components (PCs). These three PCs account for $80 \%$ of the variation present in the data set. The projections of each map onto the first three PCs are shown vectorially in Fig. 6,

Inspection of Figure 6 reveals that PC 1 is most strongly correlated with all maps. It represents a mix of the ${ }^{13} \mathrm{CO}$ and $\mathrm{HCN}$ maps, implying that PC 1 is a good density-weighted column density map of the low excitation molecules in Maffei 2. This was also found in IC 342; however, here ${ }^{13} \mathrm{CO}$ projects along PC 1 more closely than $\mathrm{HCN}$, hence PC 1 is nearer to a pure column density map than its analogue in IC 342. All maps except the low $\mathrm{S} / \mathrm{N}$ ratio $\mathrm{C}^{34} \mathrm{~S}$ show significant correlation with PC1.

PC 2 characterizes the dominant variation seen once the distribution of column density has been accounted for. PC 2 distinguishes two major distinct groups of molecules, those peaking towards the norther star forming region and those peaking towards the southern nu- clear bar end. Species with large positive projections on $\mathrm{PC} 2$ (star formation peakers) include $\mathrm{HCO}^{+}, \mathrm{HCN}$, $\mathrm{HNC}, \mathrm{C}_{2} \mathrm{H}$ and of course $3 \mathrm{~mm}$ continuum, which is freefree emission. Species with large negative projections (southern bar peakers) include $\mathrm{HNCO}, \mathrm{CH}_{3} \mathrm{OH}$ and to a lesser extent $\mathrm{N}_{2} \mathrm{H}^{+}$and $\mathrm{SiO}$. PC 3 has a quadrupolar morphology dominated by $\mathrm{SiO}$ and $\mathrm{C}^{34} \mathrm{~S}$. PC 4 and beyond are at best marginally significant so we do not discuss them in detail.

The correlation matrix resulting from the PCA (Table 1) shows in more detail the correlations between various species. The following trends are noted.

(1) $\mathrm{HCN}, \mathrm{HNC}, \mathrm{HCO}^{+}$and $3 \mathrm{~mm}$ continuum are all extremely tightly correlated, indicating a close association with massive star formation. At the resolution of the $\mathrm{PCA} \mathrm{HCO}^{+}$and $\mathrm{HCN}$ are effectively perfectly correlated. At three times higher spatial resolution subtle differences become apparent (Fig. 7). $\mathrm{HCN}$ is the map most tightly correlated with the $3 \mathrm{~mm}$ continuum. Therefore, the (massive) star formation rate versus HCN line luminosity relation seen in single-dish surveys (e.g. Gao \& Solomon 2004) persists to at least 100 pc scales in Maffei 2, as noted in (MTH08). However, the correlation between $\mathrm{HC}_{3} \mathrm{~N}(10-9)$ and $3 \mathrm{~mm}$ continuum is weaker than found for IC 342 .

(2) $\mathrm{CH}_{3} \mathrm{OH}$ and $\mathrm{HNCO}$ are very well correlated with each other and are strongly anti-correlated with the massive star formation. This leaves little doubt that on these GMC spatial scales the chemistries of these two species are either directly coupled or are mutually coupled to a specific chemical mechanism. Moreover these species show little correlation with $3 \mathrm{~mm}$ continuum and hence star formation is not relevant to establishing the morphologies of these species. Nor are these species correlated with the standard dense gas tracers, $\mathrm{HCN}, \mathrm{HCO}^{+}$ and $\mathrm{HC}_{3} \mathrm{~N}$. Fig. 8 displays the position-velocity diagrams of ${ }^{13} \mathrm{CO}, \mathrm{C}_{2} \mathrm{H}$ and HNCO. The strong differences between the starburst tracers and $\mathrm{CH}_{3} \mathrm{OH}$ or $\mathrm{HNCO}$ carry over to the kinematics. The 'parallelogram' originating from the central ring is nearly absent in $\mathrm{CH}_{3} \mathrm{OH}$ and $\mathrm{HNCO}$ but remains pronounced in $\mathrm{C}_{2} \mathrm{H}$.

(3) $\mathrm{SiO}$ and especially $\mathrm{C}^{34} \mathrm{~S}$ are less tightly correlated with the other species. This may in part be due to their low $\mathrm{S} / \mathrm{N}$ ratio and that some of the structure that appears in these maps are noise. However $\mathrm{C}^{34} \mathrm{~S}$ was also anomalous in IC 342 (MT05).

\section{DISCUSSION}

The pronounced variations in spatial morphology observed for the different molecules across the central half kiloparsec of Maffei 2 are caused by either differences in emissivity (excitation) or abundances (chemistry). In this section we discuss the possible influences on the molecular emission that could lead to the observed variations.

\subsection{The Dense Gas Component: Diagnostic Ratios}

The $\mathrm{CO} / \mathrm{HCN}$ ratio is widely used to study the relative fraction of dense gas in galaxies. MTH08 used the $\mathrm{CO} / \mathrm{HCN}$ ratio to identify the sites of high dense gas fraction and concluded that the $x_{1}-x_{2}$ orbital intersections and the eastern (star forming) portion of the central ring are the locations of the high dense gas fraction. The 
fraction of dense gas drops towards the western ring and moving out along the bar arms.

To study the nature of the dense component directly, line ratios between dense gas tracers are required, since CO does not trace dense gas. Line ratios between bright, high critical density species, such as $\mathrm{HCN}, \mathrm{HCO}^{+}, \mathrm{HNC}, \mathrm{HC}_{3} \mathrm{~N}$ and $\mathrm{CS}$ are useful diagnostics of the physical and chemical properties of the dense component of the ISM. Much observational and theoretical effort has been dedicated to understanding these diagnostic species in nearby starbursts (e.g. Henkel et al. 1991; Nguven-Q-Rieu et al. 1992; Solomon. Downes, \& Radford 1992; Helfer \& Blitz 1993; Kohno et al. 2001; Aalto et al. 2002; Gao \& Solomon 2004; Imanishi et al. 2007; Baan et al. 2008; Graciá-Carpio et al. 2008; Juneau et al. 2009. Bavet et al. 2009; Lindberg et al. 2011; Aladro et al. 2011a). Unfortunately interpreting these results can be difficult since most of these studies have been done at low spatial resolution, either with single-dish telescopes or in distant sources. These ratios mix many interstellar environments into one beam.

Now that we have access to an inventory of dense gas tracers at $30-100 \mathrm{pc}$ resolution, we investigate changes in the $\mathrm{HCN} / \mathrm{HCO}^{+}, \mathrm{HCN} / \mathrm{HNC}, \mathrm{HCO}^{+} / \mathrm{N}_{2} \mathrm{H}^{+}$, $\mathrm{HNC} / \mathrm{HCO}^{+}$and $\mathrm{HCN} / \mathrm{CS}$ line ratios on GMC scales across the nucleus of Maffei 2. Fig. 7 displays four of these line ratios. Line ratios are tabulated in Table 1 Organized variations in all ratios are observed on these scales and are discussed in terms of the $\mathrm{CO} / \mathrm{HCN}$ ratio.

\subsection{1. $\mathrm{HCN} / \mathrm{HCO}^{+}$: Tracer of Intermediate Gas Densities}

The $\mathrm{HCN} / \mathrm{HCO}^{+}$ratio relates two of the brightest lines that trace dense gas. It has become a popular diagnostic of gas with densities intermediate between $\mathrm{CO}$ and $\mathrm{HCN}$ since $\mathrm{HCO}^{+}$has a critical density nearly an order of magnitude lower than HCN (e.g., Kohno et al. 2001; Graciá-Carpio et al. 2006; Imanishi et al. 2007; Krips et al. 2008) . $\mathrm{HCO}^{+}$also has a formation route that maintains significant abundances in relatively diffuse gas (see below), and it is an ion making it more sensitive to collisions with electrons (e.g. Papadopoulos 2007) (see Table 1). As a result $\mathrm{HCO}^{+}$can remain abundant and excited in diffuse gas (Lucas \& Liszt 1996). Theoretical modeling finds that $\mathrm{HCN} / \mathrm{HCO}^{+}$is a strong function of density for a given column density in the presence of ionizing radiation, with ratios of unity for densities of $\sim 10^{5} \mathrm{~cm}^{-3}$ (e.g. Meijerink et al. 2007; Yamada et al. 2007).

There is, however, the complication of opacity. Despite its lower critical density $\mathrm{HCO}^{+}$has a higher Einstein $\mathrm{A}_{i j}$, implying higher radiative efficiencies than $\mathrm{HCN}$ (and $\mathrm{HNC}$. For a given abundance per line width, $\mathrm{HCO}^{+}$exhibits a line opacity nearly twice that of HCN and HNC. For extragalactic studies it is usually assumed that $\mathrm{HCN}$ abundances are significantly higher than $\mathrm{HCO}^{+}$. However, for the LTE abundances calculated in $\S 3.2$ and MTH08, $\mathrm{HCO}^{+}$would have approximately twice the observed abundance of $\mathrm{HCN}$, contrary to what is commonly assumed. These abundances have large uncertainties but the two lines have similar energy levels. The similarity in excitation requirements of the two lines makes it difficult to reverse this situation purely by changing gas temper- atures. If the LTE abundances are correct then $\mathrm{HCO}^{+}$ line opacities must be at least as large as the presumably large HCN line opacities. If opacities in both lines are greater than unity the diagnostic power of $\mathrm{HCN} / \mathrm{HCO}^{+}$ is limited.

Towards the nuclear bar in Maffei 2 the overall morphologies of $\mathrm{HCO}^{+}$and $\mathrm{HCN}$ are similar. However, detailed differences in the two transitions are revealed in their ratio. $\mathrm{HCN} / \mathrm{HCO}^{+}$ranges from $0.6-2.2$ over $30 \mathrm{pc}$ scales in Maffei 2. These values cover much of the range sampled in the single-dish surveys of many galaxies (Kohno et al. 2001; Graciá-Carpio et al. 2006; Krips et al. 2008). Both $\mathrm{HCN}(\mathrm{MTH} 08)$ and $\mathrm{HCO}^{+}$fall off more rapidly with galactocentric distance than does ${ }^{13} \mathrm{CO}(1-0)$. $\mathrm{HCO}^{+}$decreases more slowly than $\mathrm{HCN}$ in the north (GMC B) but more rapidly along the southern bar arm. $\mathrm{HCN}$ is elevated relative to $\mathrm{HCO}^{+}$on the active eastern side of the central ring, GMC G and a localized cloud just south of GMC F, while $\mathrm{HCO}^{+}$is enhanced, relatively speaking, in the quiescent, western ring region. We detect clouds with $\mathrm{CO} / \mathrm{HCN}$ vs. $\mathrm{HCN} / \mathrm{HCO}^{+}$values that reside in the "AGN dominated" portion of parameter space set by global single-dish studies where clearly there is no AGN (Kohno et al. 2001; Graciá-Carpio et al. 2006). Similar statements apply for "star formation dominated" parameters. As a result interpretation of singledish values must be done with great care.

The enhancement of $\mathrm{HCO}^{+}$towards the western ring and $\mathrm{HCN}$ towards the northeastern ring makes sense in the conventional critical density context, since one expects the denser gas to be more closely associated with the massive star formation. However, the elevated $\mathrm{HCN} / \mathrm{HCO}^{+}$ratio towards the southern bar end is unusual. A decreasing $\mathrm{HCN} / \mathrm{CO}$ ratio suggests the fraction of dense gas in the south is significantly lower, which should favor $\mathrm{HCO}^{+}$. That this is not seen implies some other excitation mechanism or chemistry is in play in the gas of the southwestern arm. From the distribution of shock tracers (section 4.4), GMC G must be one of the clouds most effected by shocks. It is tempting, therefore, to suggest that the southern bar end in Maffei 2 is an example of a location of elevated HCN abundances in shocks. In the Galaxy, bipolar molecular outflows do show $\mathrm{HCN}$ much more strongly elevated than $\mathrm{HCO}^{+}$ (e.g. Bachiller \& Perez Gutierrez 1997). This scenario seems less favored when the other dense gas tracers are incorporated. Towards GMC G, both the $\mathrm{HCO}^{+} / \mathrm{N}_{2} \mathrm{H}^{+}$ and $\mathrm{HCO}^{+} / \mathrm{HNC}$ ratios are anomalously low, while the $\mathrm{HCN} / \mathrm{HNC}$ ratio remains roughly constant relative to the rest of the (non-star forming) nuclear disk. This argues for a depletion/destruction of $\mathrm{HCO}^{+}$rather than an enhancement of $\mathrm{HCN}$. The decrease in $\mathrm{HCO}^{+}$intensity here may stem from the shock in a more indirect way. The passage of a shock alters the chemical state of the molecular gas, including a dramatic increase in gas-phase water, which is effective at destroying $\mathrm{HCO}^{+}$(Bergin, et al. 1998). The main difficulty with this mechanism is that $\mathrm{H}_{2} \mathrm{O}$ is also expected to destroy $\mathrm{N}_{2} \mathrm{H}^{+}$, which is not observed.

An alternate and perhaps simpler explanation for the elevated $\mathrm{HCN} / \mathrm{HCO}^{+}$ratio and the brightness of high density tracers like $\mathrm{HNCO}$ and $\mathrm{N}_{2} \mathrm{H}^{+}$at the southern bar end is that while the dense gas fraction decreases along the southern bar arm, the density of the dense 
component does not. In this context the correlation between the dense gas tracers and $\mathrm{CO} / \mathrm{HCN}$ towards the star forming ring would imply that the dense gas fraction and dense gas density are coupled there. The difference between the two environments could be a result of the different dynamical environments at each location. Towards the southern bar end one can envision a situation where the shocks (section 4.4.1) produce a low filling factor, high density component that explains the behavior of both ratios. The fact that the $\mathrm{HNCO} / \mathrm{CH}_{3} \mathrm{OH}$ ratio remains high gives additional direct evidence that densities of the shocked gas are quite high towards GMC G (section 4.4.2).

In summary, while the $\mathrm{CO} / \mathrm{HCN}$ ratio suggests a rapidly decreasing dense gas fraction along the arms (MTH08), The $\mathrm{HCN} / \mathrm{HCO}^{+}$ratio suggests that the actual density does not fall significantly. Clearly not all the properties of the dense component are traced in the $\mathrm{CO} / \mathrm{HCN}$ ratio. This further emphasizes the fact that the $C O / H C N$ measures the fraction of the gas that is dense, not necessarily the actual density of the gas. Ratios that sample only dense gas, such as $\mathrm{HCN} / \mathrm{HCO}^{+}$, are needed to determine the physical conditions of the dense component.

\subsubsection{HCN/HNC: Proposed Tracer of Warm Gas}

The HCN/HNC line ratio is another dense gas ratio of increasing popularity, in which high values indicate hot gas and/or PDR conditions. Essentially identical $\mathrm{A}_{i j}$, collision coefficients, upper energy states and frequencies make the ratio particularly insensitive to changing excitation. Dissociative recombination of $\mathrm{HCNH}^{+}$dominates the formation of both HCN and HNC in cool, dense clouds, producing both species in equal amounts (e.g. Hirota et al. 1998). So in these environments the abundances of the two species are locked together. Additionally both transitions likely have significant opacities. It is therefore not surprising that this ratio remains near unity over a wide range of conditions. When gas temperatures get large $\mathrm{HNC}$ can be converted to $\mathrm{HCN}$ via $\mathrm{HNC}+\mathrm{H} \rightarrow \mathrm{HCN}+\mathrm{H}$, while in diffuse gas / PDR-like environments, the reaction $\mathrm{N}+\mathrm{CH}_{2} \rightarrow \mathrm{HCN}+\mathrm{N}$ becomes a major additional HCN source (e.g. Schilke et al. 1992; Turner et al. 1997).

Fig. 7 (Table 1) displays the $\mathrm{HCN} / \mathrm{HNC}$ intensity ratio map. Indeed the ratio remains fairly constant across the nucleus with values of $\sim 1.1 \pm 0.3$. Variations in the ratio are only at the $30 \%$ level over most of the nucleus, with the highest values on the star forming eastern ring and south of GMC F, which suggests mild enhancement by PDRs. Ratios significantly below unity on these small spatial scales are not seen anywhere across the nucleus. Hence there is no evidence for anomalous enhancement of $\mathrm{HNC}$ relative to $\mathrm{HCN}$ that has been found in some ULIRGs (Aalto et al. 2002). In IC 342, the HCN/HNC intensity ratio is also fairly constant, with weak enhancement seen towards the PDR GMC (MT05).

In summary, at least in Maffei 2 and its less active cousin, IC 342, the $\mathrm{HCN} / \mathrm{HNC}$ line ratio has limited diagnostic power. Its diagnostic power becomes stronger for molecular gas with extreme temperatures and density, such as LIRGs and ULIRGs, where the chemistry is driven away from dissociative recombination of $\mathrm{HCNH}^{+}$.

\subsection{3. $\mathrm{HCO}^{+} / \mathrm{N}_{2} \mathrm{H}^{+}$: Tracing the Dense Gas}

Both $\mathrm{HCN}$ and $\mathrm{HCO}^{+}$(and to a lesser degree $\mathrm{HC}_{3} \mathrm{~N}$ ) are observed to maintain large abundances in PDRs, shocks and other energetic regions. By contrast, in the Galaxy $\mathrm{N}_{2} \mathrm{H}^{+}$is observed to avoid PDR regions, maintaining high abundances only in dense, quiescent, dark cloud-like conditions (Womack et al. 1992). In dense, quiescent gas both $\mathrm{N}_{2} \mathrm{H}^{+}$and $\mathrm{HCO}^{+}$form from proton transfer reactions of $\mathrm{H}_{3}^{+}$with $\mathrm{N}_{2}$ and CO, respectively:

$$
\begin{aligned}
& H_{3}^{+}+\mathrm{N}_{2} \rightarrow \mathrm{N}_{2} \mathrm{H}^{+}+\mathrm{H}_{2}, \\
& \mathrm{H}_{3}^{+}+\mathrm{CO} \rightarrow \mathrm{HCO}^{+}+\mathrm{H}_{2} .
\end{aligned}
$$

Given the reasonable assumption that the rate coefficients for these two reactions, $k 2$ and $k 3$, are approximately equal (Woodall et al. 2007), then $X\left(\mathrm{~N}_{2} \mathrm{H}_{+}\right) / X\left(\mathrm{HCO}^{+}\right) \simeq X\left(\mathrm{~N}_{2}\right) / X(\mathrm{CO})$. However, only $\mathrm{HCO}^{+}$maintains a relatively rapid formation route in diffuse or PDR gas $\left(\mathrm{C}^{+}+\mathrm{OH} \rightarrow \mathrm{CO}^{+}+\mathrm{H}\right.$ followed by $\left.\mathrm{H}_{2}+\mathrm{CO}^{+} \rightarrow \mathrm{HCO}^{+}+\mathrm{H}\right)$. No corresponding route exists for $\mathrm{N}_{2} \mathrm{H}^{+}$in PDRs (Turner 1995b). This explains the observed behavior of the $\mathrm{HCO}^{+} / \mathrm{N}_{2} \mathrm{H}^{+}$line ratio (Fig. 7 and Table 1). Towards the PDR regions associated with the active star formation sites, $\mathrm{HCO}^{+}$can maintain appreciable abundances while $\mathrm{N}_{2} \mathrm{H}^{+}$is readily destroyed. The fact that this ratio, which reflects dense gas properties, is modified by the starburst PDRs implies that towards this region (GMC E and the eastern ring) PDRs penetrate the dense gas. The strong correlation between high $\mathrm{HCO}^{+} / \mathrm{N}_{2} \mathrm{H}^{+}$ratios and $\mathrm{PDR}$ tracers like $\mathrm{C}_{2} \mathrm{H}$, suggests that this ratio may be a powerful new probe of dense PDRs in external galaxies.

Along the arms, $X\left(\mathrm{~N}_{2} \mathrm{H}^{+}\right) / X\left(\mathrm{HCO}^{+}\right)$abundances are typically $\sim 0.1-0.15$, with values reaching up to $\sim 0.33$ towards the southern bar end. If a standard $\mathrm{CO}$ abundance of $X(\mathrm{CO}) \simeq 10^{-4}$ is adopted, $X\left(\mathrm{~N}_{2}\right)$ abundances of $1-3 \times 10^{-5}$ are implied. If metallicities are solar (e.g. Anders \& Grevesse 1989) and all $\mathrm{N}$ is in the form of $\mathrm{N}_{2}$, then $X\left(\mathrm{~N}_{2}\right) \simeq 4 \times 10^{-5}$. Evidently, in the more quiescent dense clouds of the nucleus of Maffei 2, at least a quarter of all nitrogen is in molecular form. Using the above $X\left(\mathrm{~N}_{2}\right)$ estimation together with Table 1 gives $X\left(\mathrm{~N}_{2}\right) / X\left(\mathrm{~N}_{2} \mathrm{H}^{+}\right) \simeq 2 \times 10^{4}$ for these regions.

Following the analysis of MT05, $X\left(\mathrm{~N}_{2}\right) / X\left(\mathrm{~N}_{2} \mathrm{H}^{+}\right)$provides indirect constraints on the cosmic ray ionization rate, $\zeta$. Adopting $X\left(\mathrm{~N}_{2}\right) / X\left(\mathrm{~N}_{2} \mathrm{H}^{+}\right) \sim 2 \times 10^{4}$ and the extremely conservative lower limit, $X\left(e^{-}\right)=X($ ion $)$ $\geq X\left(\mathrm{~N}_{2} \mathrm{H}^{+}\right)+\mathrm{X}\left(\mathrm{HCO}^{+}\right) \geq 6-9 \times 10^{-9}$, Figure 9 of MT05 indicates $\zeta / n_{H_{2}}>10^{-21} \mathrm{~s}^{-1} \mathrm{~cm}^{3}$. Less conservatively, for $X\left(e^{-}\right) \simeq 10^{-7}$ typical of Galactic star forming regions (e.g. Caselli et al. 1998), $\zeta / n_{H_{2}}>10^{-20.5} \mathrm{~s}^{-1}$ $\mathrm{cm}^{3}$. Therefore, $\zeta$ is at least the Galactic value if the density of the gas that the $\mathrm{N}_{2} \mathrm{H}^{+}$and $\mathrm{HCO}^{+}$emission originates in is $n_{H_{2}}>10^{4} \mathrm{~cm}^{-3}$. These constraints are comparable to though somewhat stronger than observed for IC 342 .

$$
\begin{aligned}
& \text { 4.1.4. The } C S(2-1) / H C N(1-0) \text { and } C S(2-1) / C^{34} S(2-1) \\
& \text { Ratios }
\end{aligned}
$$

Kuno et al. (2008) has published a map of CS(2-1) towards the nucleus of Maffei 2 with similar spatial resolution to the data presented here. We make use of this 
map to compute the $\mathrm{CS} / \mathrm{C}^{34} \mathrm{~S}$ and $\mathrm{CS} / \mathrm{HCN}$ ratios towards the GMCs. The $\mathrm{CS} / \mathrm{HCN}$ intensity ratio is fairly constant across the nucleus except towards the southern arm (GMC G), where it is a factor of two lower. If strong chemical evolution has not altered the ${ }^{32} \mathrm{~S} /{ }^{34} \mathrm{~S}$ ratio, the $\mathrm{C}^{34} \mathrm{~S}$ emission implies the regions of high CS opacity are along the southern arm and not at the CS peak near the massive star formation. This is unexpected. For a ${ }^{32} \mathrm{~S} /{ }^{34} \mathrm{~S}$ ratio of 23 , LTE line ratios imply CS opacities of $\sim 6$ along the southern arm. Comparing the observed peak temperature of $\mathrm{C}^{34} \mathrm{~S}$ gas to optically thin emission with $\mathrm{T}_{e x}=10 \mathrm{~K}$ yields a filling factor, $f\left(C^{34} S\right) \simeq 0.018$. Given the differences in beam size this filling factor is intermediate between ${ }^{13} \mathrm{CO}$ and $\mathrm{HCN}$ (MTH08). Towards the $\mathrm{GMCs} \mathrm{D}+\mathrm{E}$ the larger $\mathrm{CS} / \mathrm{C}^{34} \mathrm{~S}$ ratio implies that CS opacities here must be less than 1.4. Since the intensity of the main isotopologue peaks towards this region, gas temperatures or filling factors are much larger here. So in order to explain the morphology of both CS and $\mathrm{C}^{34} \mathrm{~S}$, it is required that $\mathrm{CS}$ be warmer and more diffuse towards GMCs D+E when compared to the GMC G. In summary CS appears compatible with the interpretation based on $\mathrm{HCN} / \mathrm{HCO}^{+}$that the southern bar end has high density but low dense gas fraction.

\subsection{5. $\mathrm{HC}_{3} \mathrm{~N}(10-9)$}

$\mathrm{HC}_{3} \mathrm{~N}$ has a critical density that is comparable or slightly higher than HCN but very likely has low opacity due to its lower abundance. Moreover its levels are more closely spaced and so population is distributed over a wider range of levels, making any one level more sensitive to changes in excitation. $\mathrm{HC}_{3} \mathrm{~N}$ originates primarily from the arm-ring intersection regions similar to $\mathrm{HCN}$ and $\mathrm{HCO}^{+}$, although perhaps peaks slightly farther from the center of the galaxy than does HCN. Using the kinematics to further resolve the emission suggests that $\mathrm{HC}_{3} \mathrm{~N}$ is tracing the inner portion of the arm rather than the $x_{1}-x_{2}$ intersection region. Evidently, densities increase along the arms as the central ring is approached. Unlike $\mathrm{HCN}, \mathrm{HC}_{3} \mathrm{~N}$ weakens just before the strong star formation is reached at GMC D $+\mathrm{E}$ and just north of GMC F. Potentially this results from warmer temperatures depopulating the $\mathrm{J}=10$ level towards the starburst.

\subsection{PDR Chemistry and the Nuclear Starburst}

Ionizing radiation from massive stars impinging on nearby molecular clouds changes their chemical composition. Large abundances of ionized carbon in PDRs drive a rich hydrocarbon chemistry on cloud surfaces. One of the simplest species from such regions is $\mathrm{C}_{2} \mathrm{H}$. $\mathrm{C}_{2} \mathrm{H}$ is formed from rapid dissociative recombination of $\mathrm{C}_{2} \mathrm{H}_{2}^{+}$coming from reactions between $\mathrm{C}^{+}+\mathrm{CH}_{3}$ (Wootten et al. 1980; Sternberg \& Dalgarno 1995). $C_{2} H$ chemistry is particularly closely tied to ionized carbon and is an excellent probe of these PDRs. Its usefulness as an extragalactic PDR tracer was demonstrated in MT05.

After accounting for opacity effects (see 33.3 for $\mathrm{C}_{2} \mathrm{H}\left(1-0 ; \frac{1}{2}-\frac{1}{2}\right)$ for an optically thin $\mathrm{C}_{2} \mathrm{H}$ transition) $\mathrm{C}_{2} \mathrm{H}$ pervades much of the molecular clouds in the northern star forming region. Lower level $\mathrm{C}_{2} \mathrm{H}$ emission extends over the rest of the nucleus but avoids the cloud cores, instead favoring the side of the dense cloud facing star formation (traced here by $2 \mathrm{~cm}$ radio continuum) (see Fig. 9). This is particularly clearly seen west of GMC F and northeast of GMC G.

As discussed in 4.1.3. the dramatic increase in the $\mathrm{HCO}^{+} / \mathrm{N}_{2} \mathrm{H}^{+}$line ratio across the central ring shows not only that PDRs are prevalent near the young, star forming region but that the PDRs penetrate much of the dense gas there. Given the distinct region of altered chemistry, constraints can be placed on the range of influence of the current burst of star formation. For the northern star forming region, the region of elevated $\mathrm{HCO}^{+} / \mathrm{N}_{2} \mathrm{H}^{+}$ratio is $80 \mathrm{pc}$ diameter in extent. We assume that the volume of the region emitting strong PDR emission reflects the volume of molecular gas engulfed within HII regions that have expanded into the ambient medium due to over-pressure. This should be reasonable on GMC scales. For an ionized gas sound speed of 10 $\mathrm{km} \mathrm{s}^{-1}, \mathrm{~N}_{\text {Lyc }}=5 \times 10^{51} \mathrm{~s}^{-1}$ and a uniform density of $n_{H_{2}} \simeq 10^{2.75} \mathrm{~cm}^{-3}$ typical of the central ring (MTH08), a standard expanding HII region would reach a radius of $40 \mathrm{pc}$ in $\sim 7 \mathrm{Myr}$. While this analysis is sensitive to the exact density distribution, it is interesting that this timescale for dynamical evolution of GMCs due to pressure is an order of magnitude longer than characteristic chemical timescales and consistent with the timescale of massive star evolution.

\subsection{The Molecular Outflow along the Minor Axis in PDR Gas}

$\mathrm{C}_{2} \mathrm{H}$ emission appears to trace a molecular outflow along the minor axis (Fig. 9). The northwest extension of this bipolar flow is redshifted by $\sim 15 \mathrm{~km} \mathrm{~s}^{-1}$ and the southeast blob blueshifted by $\sim-15 \mathrm{~km} \mathrm{~s}^{-1}$ with respect to the velocity of the corresponding in-plane material. These velocity shifts have the correct sign for outflow given Maffei 2's geometry (northern arm being nearer to us, and the arms trailing). There is tentative evidence for the flow in $\mathrm{HNC}$ and $\mathrm{SiO}$ (and possibly $\mathrm{CO}(1-0)$; MTH08) but the molecular outflow is not obvious in any of the other species. This is understandable given the strength of $\mathrm{C}_{2} \mathrm{H}$ in the outflow reflects its relatively low critical density and its ability to maintain high abundances in the presence of ionizing radiation.

The molecular outflow in Maffei 2 does not appear to follow the distribution of ionized gas traced by $2 \mathrm{~cm}$. This is different from what is seen in the much more pronounced outflow in M82 (Walter et al. 2002). The $2 \mathrm{~cm}$ radio continuum emission appears to trace the walls of Maffei 2's outflow (Fig. 9, Turner \& Ho 1994). The $\mathrm{C}_{2} \mathrm{H}$ emission must trace entrained molecular material being driven out directly. The explanation for the $2 \mathrm{~cm}$ emission along the edges remains unclear but may be due to a path length effect of a cone of ionized gas surrounding the molecular flow. Since evidence is seen for a small minor axis outflow it is possible to obtain a second estimate of the characteristic age by assuming that the gas traced in $\mathrm{C}_{2} \mathrm{H}$ is free to flow ballistically along the minor axis. The extent of the outflow is \pm 200 pc. For an inclination angle of $67^{\circ}$ and the measured line of sight velocity, the time of flight corresponds to $6 \mathrm{Myr}$. This is a very young outflow. The agreement with the characteristic in-plane timescale is excellent, suggesting that the current starburst may have an age of $\tau \sim 6-7$ Myr. 


\subsection{Shocks and Gas-Grain Chemistry: $\mathrm{CH}_{3} \mathrm{OH}, \mathrm{HNCO}$ and $\mathrm{SiO}$}

Elevated gas-phase abundances of species whose formation is sensitive to the grain processing, such as $\mathrm{CH}_{3} \mathrm{OH}$, $\mathrm{HNCO}$, or $\mathrm{SiO}$, are important probes of hot core gas and shocks. As noted in MT05, and illustrated even more clearly here, these species do not trace hot cores. Typical Galactic hot cores, even significant collections of them cannot produce the required emission necessary to explain the intensities on these large scales. Moreover, we detect no spatial connection between $\mathrm{CH}_{3} \mathrm{OH}$ and $\mathrm{HNCO}$, and star formation, if anything, we see an anticorrelation. We can, therefore, safely conclude that the above three species are not tracing hot core emission or outflows from young stellar objects but must originate in large scale shocks. Inter-comparisons can be used to identify the responsible shock source (MT05; Requena-Torres et al. 2006; Rodriguez-Fernandez et al. 2006; Martín et al. 2008) and place constraints on shock properties such as shock strength, type of shock and liberated grain / mantle composition (Usero et al. 2006).

In this dataset three shock tracers, $\mathrm{CH}_{3} \mathrm{OH}, \mathrm{HNCO}$ and $\mathrm{SiO}$, are mapped. Each is sensitive to somewhat different shock properties. It is expected that elevated gas-phase $\mathrm{SiO}$ results from release of Si from destruction of silicate grain cores (e.g. Martín-Pintado et al. 1992). That the grain core must be disrupted requires 'strong' shocks capable of sputtering grain cores. High observed abundances of gas-phase $\mathrm{CH}_{3} \mathrm{OH}$ likely come from direct liberation of $\mathrm{CH}_{3} \mathrm{OH}$ that has formed on grain surfaces by complete hydrogenation of CO (e.g. Millar et al. 1991; Bachiller et al. 1995). Liberation of mantles does not require shocks as strong as those that effect the grain core. Thus the relative enhancement of $\mathrm{SiO}$ versus $\mathrm{CH}_{3} \mathrm{OH}$ will depend on the strength of the shock. Grain core destruction requires shock velocities of $\gtrsim 25 \mathrm{~km} \mathrm{~s}^{-1}$ whereas shock velocities of only $\gtrsim 10-15 \mathrm{~km} \mathrm{~s}^{-1}$ are required for significantly elevated gas-phase $\mathrm{CH}_{3} \mathrm{OH}$ abundances (e.g. Caselli et al. 1997; Bergin, et al. 1998). It is reasonably well established that HNCO is a mantle species along with $\mathrm{CH}_{3} \mathrm{OH}$ (MT05; Rodríguez-Fernández et al. 2010). But its formation mechanism remains unclear. The most commonly proposed mechanism is partial hydrogenation of OCN on grain surfaces followed by direct liberation (e.g. Hasegawa \& Herbst 1993). Other suggested mechanisms include acid-base reactions between $\mathrm{CO}$ and $\mathrm{NH}_{3}$ and UV photolysis (Schutte \& Greenberg 1997; Keane et al. 2001). Thus if HNCO forms on grain mantles and is liberated directly then its unsaturated nature implies that it originates in grain mantles with different chemical composition from $\mathrm{CH}_{3} \mathrm{OH}$ (however see Tideswell et al. (2010) for a possible formation mechanism in more saturated ices). Unsaturated ices are favored when gas accretes in atomic hydrogen-poor environment whereas saturated ices favor atomic hydrogen-rich accretion (e.g. Charnley et al. 1997; Watanabe \& Kouchi 2002). Hydrogen-poor conditions reflect accretion at high densities when almost all hydrogen is in molecular form. If it is assumed that the shock simply injects molecules into the gas phase, the $\mathrm{HNCO} / \mathrm{CH}_{3} \mathrm{OH}$ abundance ratio is then sensitive to gas physical conditions during ice mantle formation.

Chemistry is not the only effect that influences the relative abundance of these three species. Excitation is also important. The excitation requirements of $\mathrm{SiO}(2$ 1), $\mathrm{CH}_{3} \mathrm{OH}\left(2_{k}-1_{k}\right)$ and $\mathrm{HNCO}\left(5_{05}-4_{04}\right)$ transitions differ (see Table 1). The critical density of the HNCO transition is an order of magnitude higher than $\mathrm{SiO}$ and $\mathrm{CH}_{3} \mathrm{OH}$. Furthermore the energy of the upper state in $\operatorname{HNCO}\left(5_{05}-4_{04}\right)$ is more than twice that of $\mathrm{SiO}$ and higher than two of the three main transitions that make up the $\mathrm{CH}_{3} \mathrm{OH}$ line. Therefore, $\mathrm{CH}_{3} \mathrm{OH}$ and $\mathrm{SiO}$ lines will be favored over $\mathrm{HNCO}$ in lower density gas. Also in the presence of ionizing radiation each species dissociates at different rates. $\mathrm{HNCO}$ is photodissociated at a rate twice that of $\mathrm{CH}_{3} \mathrm{OH}$ and $\sim 30$ times that of $\mathrm{SiO}$ (Roberge et al. 1991; Sternberg \& Dalgarno 1995).

Thus considering the distribution of $\mathrm{CH}_{3} \mathrm{OH}, \mathrm{HNCO}$, and $\mathrm{SiO}$ can give insights into both the chemical and physical conditions present in the shocked gas and potentially tell us about nature of the shocks themselves. Below we discuss the overall structure of the shock tracers in the context of nuclear bar models, then we analyze the subtle differences between each shock tracer to further constrain shock properties.

\subsubsection{Overall Shock Tracer Morphology and Bar Structure}

In Maffei 2 the outer bar arms dominate the overall distribution of the shock tracers $\mathrm{CH}_{3} \mathrm{OH}, \mathrm{HNCO}$, and $\mathrm{SiO}$, whereas emission from the star forming ring is minimal. This is very different from the morphology of $\mathrm{CO}$, HCN and the PDR tracers. The bar ends are especially bright in the shock tracers. (The faintness of shock tracers along the northern arm is presumably due to the fact that there is much less molecular gas there, particularly at what would be the location of the northern bar end.) It is perhaps surprising that it is the bar ends and not the inner arms that have the largest fraction of the gas influenced by shocks. This provides constraints on the nature of gas flows in Maffei 2 .

Different models exist for describing the structure of gas bars, each predicting different shock strengths and locations. The models fall into two broad classes, orbitcrowding models and hydrodynamical flows. Orbitcrowding models treat the gas as distinct clouds orbiting on essentially ballistic orbits set up by the potential (e.g. Combes \& Gerin 1985; Byrd et al. 1994). The arm morphology of the bar is then determined by where orbits converge. In hydrodynamical models the gas is treated as a fluid that responds to the stellar potential and is susceptible to hydrodynamical forces such as pressure and viscosity. In such models, gas flows along streamlines with shocks occurring where the streamlines collide (e.g. Athanassoula 1992; Piner et al. 1995). Both models predict similar morphological distributions but rather distinct shock properties (e.g. Regan et al. 1999). In orbit-crowding models the bar arms result from molecular clouds moving along converging orbits. In such models only weak shocks are expected because the bulk of the velocities are parallel to both clouds (with the possible exception of arm / nuclear ring intersections). Moreover such models do not isolate the bar ends as locations of strongly convergent orbits and so it is not expected that these location would be sites of vigorous shock activity.

Hydrodynamical models, on the other hand, show gas flow streamlines that change directions dramatically. The places where flows converge are accompanied by 
strong changes in velocity, and hence strong shocks. In hydro models, gas drifts slowly inward along bar-aligned $x_{1}$ stellar orbits (or in the case of Maffei 2, from radial inflow along the outer bar) until the gas reaches the innermost, cuspy $x_{1}$ orbit (of the nuclear bar). The cusps of these orbits lie at the bar ends. There the gas is compressed, shocks and plunges nearly radially inward along the bar arm until it reaches the much further in, perpendicular to the bar $x_{2}$ orbitals, where it is again shocked, loses angular momentum and transitions onto the $x_{2}$ orbitals. Some high angular momentum gas 'sprays' off the inner $x_{2}$ orbits, swinging out and colliding with the arm gas flow (e.g. Athanassoula 1992; Piner et al. 1995; Kim et al. 2011). The amount, location and relative velocity of gas in the spray region depends on bar parameters.

In summary, the strength of the emission from the shock tracers $\mathrm{CH}_{3} \mathrm{OH}, \mathrm{HNCO}$, and $\mathrm{SiO}$ along the bright bar ends favors the hydrodynamical bar models, as orbitcrowding models do not predict strong cloud collisions at the bar end whereas they are sites of shocks in hydrodynamical models.

\subsubsection{Relative Shock Tracer Morphologies and Bar Properties}

What about changes in shock properties across the nuclear bar? Fig. 10 displays the $\mathrm{HNCO} / \mathrm{CH}_{3} \mathrm{OH}$, $\mathrm{SiO} / \mathrm{CH}_{3} \mathrm{OH}$ and $\mathrm{SiO} / \mathrm{HNCO}$ peak temperature ratios smoothed to $8^{\prime \prime}$ and sampled at the GMCs. Given the significant difference in the native resolution of the three datasets and the fact that they were obtained with two different interferometers, only the clearest trends are discussed.

As demonstrated by the principal component analysis, the morphologies of the three species are strongly correlated, implying that we have a robust measure of the locations of shocked gas. So similar are the maps that only very subtle differences between $\mathrm{HNCO}, \mathrm{CH}_{3} \mathrm{OH}$ and $\mathrm{SiO}$ appear, suggesting that shock conditions are uniform across the nucleus. With the possible exception of the uncertain $\mathrm{SiO}$ ratios towards GMC B, the arm clouds $(\mathrm{C}$, $\mathrm{D}, \mathrm{F}, \mathrm{G})$ have effectively constant $\mathrm{HNCO} / \mathrm{CH}_{3} \mathrm{OH}(\sim$ $1.1 \pm 0.2), \mathrm{SiO} / \mathrm{CH}_{3} \mathrm{OH}(\sim 0.20 \pm 0.02)$ and $\mathrm{SiO} / \mathrm{HNCO}$ $(\sim 0.19 \pm 0.03)$ ratios. Only towards GMC E and the off arm spray regions (GMC H and just south of the western ring) are different ratios observed. Towards GMC $\mathrm{E}$ (the northern star forming region), $\mathrm{HNCO} / \mathrm{CH}_{3} \mathrm{OH}$ is lower and the $\mathrm{SiO}$ ratios are higher relative to the rest of the arm. In the off arm regions $\mathrm{HNCO} / \mathrm{CH}_{3} \mathrm{OH}$ ratios are depressed while the $\mathrm{SiO} / \mathrm{CH}_{3} \mathrm{OH}$ ratio is elevated and $\mathrm{SiO} / \mathrm{HNCO}$ are elevated (spray region) or flat $(\mathrm{GMC} \mathrm{H})$.

From these ratios, three main points are concluded. 1) While GMC G (the bar end) is the brightest source in the nuclear bar, indicating that the largest fraction of gas along this line of sight is shocked, evidently shock strengths are not enhanced here. SiO to mantle species have the same basic ratio at the bar end as it has over the rest of the bar.

2) Towards GMC E, the decreased intensity of HNCO relative to $\mathrm{CH}_{3} \mathrm{OH}$ and $\mathrm{SiO}$ is consistent with what is expected from photodissociation. From section 4.2, we know that the dense gas here is permeated by UV photons for the young star formation. The shock tracer ratios further confirm this. Because of this, the absence (or faintness) of shock tracers towards the central ring and in particular the northern $x_{1}-x_{2}$ orbit intersection cannot be taken as evidence of absence of shocks here.

3) The spray region upstream (counterclockwise) from the main bar arms favor $\mathrm{CH}_{3} \mathrm{OH}$ and to a lesser degree $\mathrm{SiO}$ relative to $\mathrm{HNCO}$. It is possible that because gas is more diffuse in this region grain mantles are H-richer than in the denser arm. However, we favor a simpler explanation associated with changing excitation. The spray regions have lower density hence the higher critical density HNCO transition is expected to be weaker relative to the lower critical density, $\mathrm{CH}_{3} \mathrm{OH}$ and $\mathrm{SiO}$ transitions. That the $5_{05}-4_{04}$ transition of HNCO preferentially favors the dense clouds relative to more extended $\mathrm{CH}_{3} \mathrm{OH}$ explains why larger beam single-dish multi-line observations derive an excitation temperature for $\mathrm{HNCO}$ that is nearly a factor of $\sim 2$ larger than derived for the $\mathrm{CH}_{3} \mathrm{OH}$ transitions in Maffei 2 (Hüttemeister et al. 1997; Martín et al. 2009). Note that the excitation temperature of both species is quite low $\left(\mathrm{T}_{e x}<15 \mathrm{~K}\right.$ ), implying that the shock regions remain cool or the densities are low enough for these transitions to be sub-thermal.

In summary, we conclude that there is little evidence for strongly varying grain chemistry across the shocked regions on $8^{\prime \prime}$ scales. The small level of variation seen is consistent with changing physical conditions (radiation field and gas density).

\subsection{The Chemistry of Spiral Nuclei: Maffei 2 vs. IC 342}

The overall nuclear structures of Maffei 2 and IC 342 are similar. Both have extended bar-like arms that terminate on a central ring that is actively forming stars. The molecular gas morphology of IC 342 is consistent with that of a gaseous bar (Turner \& Hurt 1992). However, because of the very small central ring and ambiguous kinematics, other conclusions including unbarred spiral arms coupled with massive star feedback remain feasible (Schinnerer et al. 2008). On the other hand Maffei 2 is clearly a nuclear bar based on both its morphology and kinematics (e.g. MTH08; Kuno et al. 2008). Given the potential dynamical similarities of the two nuclei a comparison of the chemistry of the two can provide important insights on the generalizability of the the overall chemical picture developed in MT05.

The statistically robust principal component analysis demonstrates that the chemistry of the two nuclei are remarkably consistent. Maffei 2 can be defined chemically as a highly inclined copy of IC 342 (Fig. 11). In both systems the first principal component characterizes the density-weighted column density, and peaks along the inner arms and central ring. The second principal component separates the main chemical forces responsible for controlling observed abundances, namely shocks versus PDRs. PDR species dominate close to the young star forming region while the shock tracers peak along the arm, with preference for the cuspy bar ends. As a result, the bar driven star formation / chemical scenario outlined in MT05 must apply also to Maffei 2's nuclear bar.

However the chemical picture is not precisely identical, and these differences give added insight into the relative differences in evolutionary phase of the two nuclei. The 
main difference between the two galaxies is in the distribution of $\mathrm{N}_{2} \mathrm{H}^{+}$. In IC 342 the PDR tracers are confined, appearing to be primarily cloud surface features, lining the inner ring. Significant $\mathrm{N}_{2} \mathrm{H}^{+}$(and $\mathrm{HNCO}$ ) seen near the young star forming region in IC 342 implies that its main star forming region influences the dense gas only locally, on scales of $50 \mathrm{pc}$ or less. Whereas in Maffei 2 , the $\mathrm{N}_{2} \mathrm{H}^{+} / \mathrm{HCO}^{+}$ratio and photodissociation of $\mathrm{HNCO}$ clearly demonstrate that the star formation has begun to dramatically alter the chemical state of the dense molecular clouds in its immediate environment, not just the surfaces.

Evidently the central molecular ring in Maffei 2 is structurally different (lower clump density) and that towards GMC E star formation is somewhat more evolved than the main burst in IC 342. This is consistent with the steeper radio spectrum towards GMC E (Turner \& Ho 1994; Tsai et al. 2006). The crude estimates of timescale required to alter the chemistry of the dense component is $\sim 7$ Myrs. This is a reasonable timescale compared to stellar evolution of the burst in Maffei 2 and the kinematics of the outflow. However, this timescale is still very short; short enough that even this 'evolved' part of the burst is in its early stages. The star formation extending to the north and south of GMC E is likely as young as the embedded star formation in IC 342.

In IC 342 the peak temperature ratios between $\mathrm{SiO}$, $\mathrm{CH}_{3} \mathrm{OH}$ and $\mathrm{HNCO}$, are similar to those seen in Maffei 2. The PDR GMC in IC 342 has slightly lower $\mathrm{HNCO} / \mathrm{CH}_{3} \mathrm{OH}$ and elevated $\mathrm{SiO} / \mathrm{HNCO}$ ratios. This is exactly the behavior exhibited by the PDR-modified GMC E in Maffei 2, lending strong support for the influence of photodissociation on shock tracer brightness. The shock chemistry dominated cloud in IC 342 has slightly elevated HNCO relative to the other species, making its set of ratios a match within the uncertainties of GMC G. This agreement suggests that both the grain composition and shock properties are the same for these structurally similar locales. However, since brightnesses are sensitive to radiative transfer effects, one must be careful over-interpreting ratios if gas excitation is changing rapidly. Maffei 2 and IC 342 do have rather similar $\mathrm{CO}$ excitation conditions (Meier et al. 2000; Meier \& Turner 2001; MTH08).

In summary, we find that when the dynamical environments are similar, as they are in IC 342 and Maffei 2, a consistent chemical picture begins to emerge. Gas chemistry is confirmed as a powerful method for isolating the physical mechanisms that influence gas conditions in spiral nuclei. In future papers in this series, we will broaden the range of galactic environments studied to assess how far chemistry can be pushed as a diagnostic of sub-beam physics.

\section{CONCLUSIONS}

This paper presents $2^{\prime \prime}-10^{\prime \prime}$ imaging of transitions from nine separate molecules across the nuclear bar in Maffei 2 obtained with the BIMA and OVRO millimeter interferometers. The results are compared with the first paper in the series on IC 342 to test the generalizability of the chemistry discovered there and to constrain differences in structure between the two nuclei. Our main conclusions are as follows:
1. Morphologies of the observed transitions exhibit both dramatic (e.g., $\mathrm{C}_{2} \mathrm{H}$ vs. $\mathrm{CH}_{3} \mathrm{OH}$ ) and subtle (e.g., $\mathrm{HCN}$ vs. $\mathrm{HCO}^{+}$) spatial differences indicative of the varying dominance of shocks vs. radiation fields as drivers of the chemistry in individual clouds.

2. A principal component analysis reveals that $\mathrm{HCN}$, $\mathrm{HNC}, \mathrm{HCO}^{+}$and $3 \mathrm{~mm}$ continuum are all extremely tightly correlated, indicating a direct connection to massive star formation.

3. $\mathrm{CH}_{3} \mathrm{OH}$ and $\mathrm{HNCO}$ are tightly correlated with each other and are strongly anti-correlated with the tracers of massive star formation; based on their spatial distribution, it appears that these two molecules are formed by liberation of grain mantles in the presence of bar shocks.

4. Variations in the ratios amongst the popular $\lambda \sim 3$ mm dense gas tracers, $\mathrm{HCN}, \mathrm{HCO}^{+}, \mathrm{HNC}, \mathrm{CS}$ and $\mathrm{HC}_{3} \mathrm{~N}$, are at or less than a factor of three, unlike the order of magnitude variations exhibited by the chemistry-sensitive molecules. This indicates that variations in excitation in the cool, dense gas are much less dramatic than variations in dense gas chemical conditions.

5. Localized variations in the ratios $\mathrm{HCN} / \mathrm{HCO}^{+}$, $\mathrm{HCN} / \mathrm{HNC}, \mathrm{HCN} / \mathrm{CS}$ and $\mathrm{HCN} / \mathrm{CO}$ can be explained by variations in density. The dense gas ratios indicate that the highest density gas is found at the $x_{1}-x_{2}$ orbit intersections, the lowest density gas is along the non star-forming western portion of the ring, and intermediate density gas is found near the star formation. Although high density gas is found throughout the full extent of the nuclear bar arms, the dense gas fraction falls at larger radii from the nucleus.

6. There is no evidence for X-ray dissociation regions or AGN influences on the gas in the center of Maffei 2 , specifically, as traced by the $\mathrm{HCN} / \mathrm{HCO}^{+}$ratio.

7. The strong correlation between high $\mathrm{HCO}^{+} / \mathrm{N}_{2} \mathrm{H}^{+}$ ratios and the $\mathrm{PDR}$ tracer molecule, $\mathrm{C}_{2} \mathrm{H}$, suggests that this ratio may be a powerful new probe of dense PDRs in external galaxies. Given the high critical density of both $\mathrm{HCO}^{+}$and $\mathrm{N}_{2} \mathrm{H}^{+}$in comparison to $\mathrm{C}_{2} \mathrm{H}$, the elevated ratio demonstrates that PDR gas towards the strong star forming regions permeate the dense clumps. When combined with the morphology of $\mathrm{C}_{2} \mathrm{H}$ this ratio may provide useful insights into the relative density of PDR gas.

8. $\mathrm{C}_{2} \mathrm{H}$ emission reveals a molecular outflow along the minor axis that has entrained molecular gas. Wrapped around the outside of this outflow is ionized gas traced in $2 \mathrm{~cm}$ radio continuum. Assuming ballistic motion, the morphology and kinematics are consistent with a very young age of 6-7 Myrs for the outflow.

9. The bar induced orbital shocks in Maffei 2 are traced in three species, $\mathrm{HNCO}, \mathrm{CH}_{3} \mathrm{OH}$ and $\mathrm{SiO}$. 
All three species support the conclusion that the nuclear bar end is the site of the largest fraction of shocked gas. That this location exhibits such strong shock properties argues in favor of hydrodynamical bar models.
We appreciate the support and assistance of the faculty, staff and postdocs at BIMA and OVRO during the observations. We L. E. Snyder and E. C. Sutton for helpful discussions. The referee is acknowledged for a thorough and very helpful report. DSM acknowledges support from NSF AST-0506669, AST-1009620 and the National Radio Astronomy Observatory which is operated by Associated Universities, Inc., under cooperative agreement with the National Science Foundation. Additional support for this work is provided by NSF grant AST-0506469 to JLT.

\section{REFERENCES}

Aalto, S., Polatidis, A. G., Hüttemeister, S. \& Curran, S. J. 2002, A\&A, 381, 783

Aladro, R., Martín-Pintado, J., Martín, S., Mauersberger, R., \& Bayet, E. 2011a, A\&A, 525, A89

Aladro, R., Martín, S., Martín-Pintado, J., Mauersberger, R., Henkel, C. Ochaña, B \& Amo-Baladrón, A 2011b, arXiv:1109.6236

Anders, E. \& Grevesse, N. 1989, Geochim. Cosmochim. Acta, 53, 197

Athanassoula, E. 1992, MNRAS, 259, 328

Baan, W. A., Henkel, C., Loenen, A. F., Baudry, A., \& Wiklind, T. 2008, A\&A, 477, 747

Bachiller, R., Liechti, S., Walmsley, C. M., \& Colomer, F. 1995, A\&A, 295, L51

Bachiller, R. \& Perez Gutierrez, M. 1997, ApJ, 487, L93

Bayet, E., Aladro, R., Martín, S., Viti, S., \& Martín-Pintado, J. 2009, ApJ, 707, 126

Benson, P. J., Caselli, P., \& Myers, P. C. 1998, ApJ, 506, 743

Bergin, E. A., Melnick, G. J., \& Neufeld, D. A. 1998, ApJ, 499, 777

Blake, G. A., Sutton, E. C., Masson, C. R. \& Phillips, T. G. 1987, ApJ, 315, 621

Byrd, G., Rautiainen, P., Salo, H., Buta, R., \& Crocher, D. A. 1994, AJ, 108, 476

Caselli, P., Hartquist, T. W., \& Havnes, O. 1997, A\&A, 322, 296

Caselli, P., Walmsley, C. M., Terzieva, R., \& Herbst, E. 1998, ApJ, 499, 234

Charnley, S. B., Tielens, A. G. G. M., \& Rodgers, S. D. 1997, ApJ, 482, L203

Chin, Y.-N., Henkel, C., Whiteoak, J. B., Langer, N., \& Churchwell, E. B. 1996, A\&A, 305, 960

Chung, H. S., Osamu, K., \& Masaki, M. 1991, JKAS, 24, 217

Combes, F., \& Gerin, M. 1985, A\&A, 150, 327

Costagliola, F. et al. 2011, A\&A, 528, A30

Crosthwaite, L. P., Turner, J. L., Hurt, R. L., et al. 2001, AJ, 122,797

Dayou, F., \& Balança, C. 2006, A\&A, 459, 297

de Vicente, P., Martín-Pintado, J., Neri, R., \& Colom, P. 2000, A\&A, 361, 1058

Dickinson, A. S., Phillips, T. G., Goldsmith, P. F., Percival, I. C. \& Richards, D. 1977, A\&A, 54, 645

Drdla, K., Knapp, G. R., \& van Dishoeck, E. F. 1989, ApJ, 345, 815

Everitt, B. S., \& Dunn, G. 2001, Applied Multivariate Data Analysis (London:Oxford Univ. Press), 48

Fingerhut, R. L., Lee, H., McCall, M. L., \& Richer, M. G. 2007, ApJ, 655, 814

Flower, D. R. 1999, MNRAS, 305, 651

Gao, Y. \& Solomon, P. M. 2004, ApJ, 606, 271

Garcia-Burillo, S., Martín-Pintado, J., Fuente, A. \& Neri, R. 2000, A\&A, 355, 499

García-Burillo, S., Martín-Pintado, J., Fuente, A., \& Neri, R. 2001, ApJ, 563, L27

Graciá-Carpio, J., García-Burillo, S., Planesas, P., \& Colina, L. 2006, ApJ, 640, L135

Graciá-Carpio, J., García-Burillo, S., Planesas, P., Fuente, A., \& Usero, A. 2008, A\&A, 479, 703

Greaves, J. S., Ohishi, M., \& Nyman, L.-A. 1996, A\&A, 307, 898

Green, S. 1975, ApJ, 201, 366

Green, S. 1986, NASA Tech. Memo., NASA TM 87791
Green, S. \& Chapman, S. 1978, ApJS, 37, 169

Green, S. \& Thaddeus, P. 1974, ApJ, 191, 653

Hasegawa, T. I., \& Herbst, E. 1993, MNRAS, 263, 589

Helfer, T. T. \& Blitz, L. 1993, ApJ, 419, 86

Henkel, C., Baan, W. A. \& Mauersberger, R. 1991, A\&AR, 347

Heyer, M. H., \& Schloerb, F. P. 1997, ApJ, 475, 173

Hirota, T., Yamamoto, S., Mikami, H., \& Ohishi, M. 1998, ApJ, 503,717

Huggins, P. J., Carlson, W. J., \& Kinney, A. L. 1984, A\&A, 133, 347

Hurt, R. L., Merrill, K. M., Gatley, I., \& Turner, J. L. 1993, AJ, 105,121

Hurt, R. L., \& Turner, J. L. 1991, ApJ, 377, 434

Hurt, R. L., Turner, J. L., \& Ho, P. T. P. 1996, ApJ, 466, 135

Hüttemeister, S., Mauersberger, R. \& Henkel, C. 1997, A\&A, 326, 59

Imanishi, M., Nakanishi, K., Tamura, Y., Oi, N., \& Kohno, K. 2007, AJ, 134, 2366

Ishiguro, M., Kawabe, R., Morita, K.-I., et al. 1989, ApJ, 344, 763

Juneau, S., Narayanan, D. T., Moustakas, J., Shirely, Y. L.,

Bussman, R. S., Kennicutt, R. C., Jr., \& Vanden Bout, P. A. 2009, ApJ, 707, 1217

Kalenskii, S. V., Dzura, A. M., Booth, R. S., Winnberg, A., \& Alakoz, A. V. 1997, A\&A, 321, 311

Kalenskii, S. V. \& Sobolev, A. M. 1994, AstL, 20, 91

Keane, J. V., Tielens, A. G. G. M., Boogert, A. C. A., Schutte, W. A., \& Whittet, D. C. B. 2001, A\&A, 376, 254

Kim, S. S., Saitoh, T. R., Jeon, M., Figer, D., Merritt, D. \& Wada, K. 2011, ApJ, 735, L11

Kohno, K., Matsushita, S., Vila-Vilaró, B., Okumura, S. K., Shibatsuka, T., Okiura, M, Ishizuki, S. Kawabe, R. 2001, The Central Kiloparsec of Starbursts and AGN: The La Palma Connection, 249, 672

Krips, M., Neri, R., García-Burillo, S., Martín, S., Combes, F., Graciá-Carpio, J. \& Eckart, A. 2008, ApJ, 677, 262

Krzanowski, W. J. 1988, Principles of Multivariate Analysis (Oxford: Clarendon)

Kuno, N., Nakanishi, K., Sorai, K., \& Shibatsuka, T. 2008, PASJ, 60,475

Lapinov, A. V., Schilke, P., Juvela, M., \& Zinchenko, I. I. 1998, A\&A, 336, 1007

Lindberg, J. E., Aalto, S., Costagliola, F., Pérez-Beaupuits, J-P., Monje, R. \& Muller, S. 2011, A\&A, 527, A150

Lucas, R., \& Liszt, H. 1996, A\&A, 307, 237

Lucas, R.\& Liszt, H. S. 2000, A\&A, 358, 1069

Lucas, R., \& Liszt, H. S. 2002, A\&A, 384, 1054

Martín-Pintado, J., Bachiller, R. \& Fuente, A. 1992, A\&A, 254, 315

Martín, S., Martín-Pintado, J., \& Mauersberger, R. 2009, ApJ, 694,610

Martín, S., Mauersberger, R., Martín-Pintado, J., Henkel, C., \& García-Burillo, S. 2006, ApJS, 164, 450

Martín, S., Requena-Torres, M. A., Martín-Pintado, J., \&

Mauersberger, R. 2008, ApJ, 678, 245

Meier, D. S. \& Turner, J. L. 2001, ApJ, 551, 687

Meier, D. S. \& Turner, J. L. 2005, ApJ, 618, 259

Meier, D. S., Turner, J. L. \& Hurt, R. L. 2000, ApJ, 531, 200

Meier, D. S., Turner, J. L., \& Hurt, R. L. 2008, ApJ, 675, 281

Meier, D. S., Turner, J. L., \& Schinnerer, E. 2011, AJ, 142, 32

Meijerink, R., Spaans, M., \& Israel, F. P. 2007, A\&A, 461, 793 
Millar, T. J., Herbst, E. \& Charnley, S. B. 1991, ApJ, 369, 147 Minier, V. \& Booth, R. S. 2002, A\&A, 387, 179

Morata, O., Estalella, R., Lopez, R., \& Planesas, P. 1997, MNRAS, 292, 120

Morris, M., Turner, B. E., Palmer, P., \& Zuckerman, B. 1976, ApJ, 205, 82

Murtagh, F., \& Heck, A. 1987, Multivariate Data Analysis (Dordrecht: D. Reidel)

Nguyen-Q-Rieu, Jackson, J. M., Henkel, C., Truong, B. \& Mauersberger, R. 1992, ApJ, 399, 521

Nummelin, A., Bergman, P., Hjalmarson, A., Friberg, P., Irvine, W. M., Millar, T. J., Ohishi, M., \& Saito, S. 2000, ApJS, 128, 213

Nyman, L.-A. 1984, A\&A, 141, 323

Padin, S., Scott, S. L., Woody, D. P., Scoville, N. Z., Seling, T. V., Finch, R. P., Ciovanine, C. J., \& Lowrance, R. P. 1991, PASP, 103, 461

Papadopoulos, P. P. 2007, ApJ, 656, 792

Pickett, H. M. , R. L. Poynter, E. A. Cohen, M. L. Delitsky, J. C. Pearson, \& H. S. P. Muller, 1998, "Submillimeter, Millimeter, and Microwave Spectral Line Catalog," J. Quant. Spectrosc. \& Rad. Transfer 60, 883-890.

Piner, B. G., Stone, J. M., \& Teuben, P. J. 1995, ApJ, 449, 508

Pirogov, L., Zinchencko, I., Lapinov, A., Myshenko, V. \& Shul'Ga, V. 1995, A\&AS, 109, 333

Pottage, J. T., Flower, D. R., \& Davis, S. L. 2004, MNRAS, 352, 39

Regan, M. W., Sheth, K., \& Vogel, S. N. 1999, ApJ, 526, 97

Requena-Torres, M. A., Martín-Pintado, J., Rodríguez-Franco, A., Martín, S., Rodríguez-Fernández. N. J. \& de Vincente, P. 2006, A\&A, 455, 971

Roberge, W. G., Jones, D., Lepp, S., \& Dalgarno, A. 1991, ApJS, 77,287

Rodriguez-Fernandez, N. J., Combes, F., Martín-Pintado, J., Wilson, T. L., \& Apponi, A. 2006, A\&A, 455, 963

Rodríguez-Fernández, N. J., Tafalla, M., Gueth, F., \& Bachiller, R. 2010, A\&A, 516, A98

Schilke, P., Walmsley, C. M., Pineau Des Forets, G., Roueff, E., Flower, D. R., \& Guilloteau, S. 1992, A\&A, 256, 595

Schinnerer, E., Böker, T., Meier, D. S., \& Calzetti, D. 2008, ApJ, 684, L21

Schutte, W. A. \& Greenberg, J. M. 1997, A\&A, 317, L43

Scoville, N. Z., Carlstrom, J., Padin, S., Sargent, A., Scott, S. \& Woody, D. 1994, Astronomy with Millimeter and Submillimeter Wave Interferometry, IAU Colloquium 140, ASP Conference Series, Vol. 59, 1994, M. Ishiguro and J. Welch, Eds., p.10

Snell, R. L., Narayanan, G., Yun, M. S., Heyer, M., Chung, A., Irvine, W. M., Erickson, N. R. \& Liu, G. 2011, AJ, 141, 38
Snyder, L. E., \& Buhl, D. 1975, ApJ, 197, 329

Solomon, P. M., Downes, D., \& Radford, S. J. E. 1992, ApJ, 387, L55

Sternberg, A. \& Dalgarno, A. 1995, ApJS, 99, 565

Tideswell, D. M., Fuller, G. A., Millar, T. J., \& Markwick, A. J. 2010, A\&A, 510, A85

Tsai, C.-W., Turner, J. L., Beck, S. C., Crosthwaite, L .P., Ho, P. T. P. \& Meier, D. S. 2006, AJ, 132, 2383

Turner, B. E. 1991, ApJS, 76, 617

Turner, B. E. 1995a, ApJ, 444, 708

Turner, B. E. 1995b, ApJ, 449, 635

Turner, B. E. 1998a, ApJ, 501, 731

Turner, B. E. 1998b, ApJ, 495, 804

Turner, B. E., Pirogov, L., \& Minh, Y. C. 1997, ApJ, 483, 235

Turner, B. E., Terzieva, R., \& Herbst, E. 1999, ApJ, 518, 694

Turner, J. L., \& Ho, P. T. P. 1983, ApJ, 268, L79

Turner, J. L., \& Ho, P. T. P. 1994, ApJ, 421, 122

Turner, J. L., \& Hurt, R. L. 1992, ApJ, 384, 72

Usero, A., García-Burillo, S., Fuente, A., Martín-Pintado, J., \& Rodríguez-Fernández, N. J. 2004, A\&A, 419, 897

Usero, A., García-Burillo, S., Martín-Pintado, J., Fuente, A., \& Neri, R. 2006, A\&A, 448, 457

Vanden Bout, P. A., Loren, R. B., Snell, R. L., \& Wootten, A. 1983, ApJ, 271, 161

van Dishoeck, E. F., \& Blake, G. A. 1998, ARA\&A, 36, 317

Wall, J. V. \& Jenkins, C. R. 2003, in "Practical Statistics for Astronomers" (Cambridge Univ. Press: Cambridge), chp. 4.5

Walter, F., Weiss, A. \& Scoville, N. 2002, ApJ, 580, L21

Wang, Y., Jaffe, D. T., Evans, N. J., Hayashi, M., Tatematsu, K., \& Zhou, S. 1993, ApJ, 419, 707

Watanabe, N., \& Kouchi, A. 2002, ApJ, 571, L173

Watt, G. D. 1983, MNRAS, 205, 321

Welch, W. J. et al. 1996, PASP, 108, 93

Womack, M., Ziurys, L. M. \& Wyckoff, S. 1992b, ApJ, 387, 417

Woodall, J., Agúndez, M., Markwick-Kemper, A. J., \& Millar,

T. J. 2007, A\&A, 466, 1197

Wootten, A., Bozyan, E. P., Garrett, D. B., Loren, R. B. \& Snell, R. L. 1980, ApJ, 239, 844

Wootten, A., Evans, N. J., Snell, R., \& Vanden Bout, P. 1978, ApJ, 225, L143

Yamada, M., Wada, K., \& Tomisaka, K. 2007, ApJ, 671, 73

Zinchenko, I, Henkel, C., \& Mao, R. Q. 2000, A\&A, 361, 1079

Ziurys, L. M., Friberg, P. \& Irvine, W. M. 1989, ApJ, 343, 201 


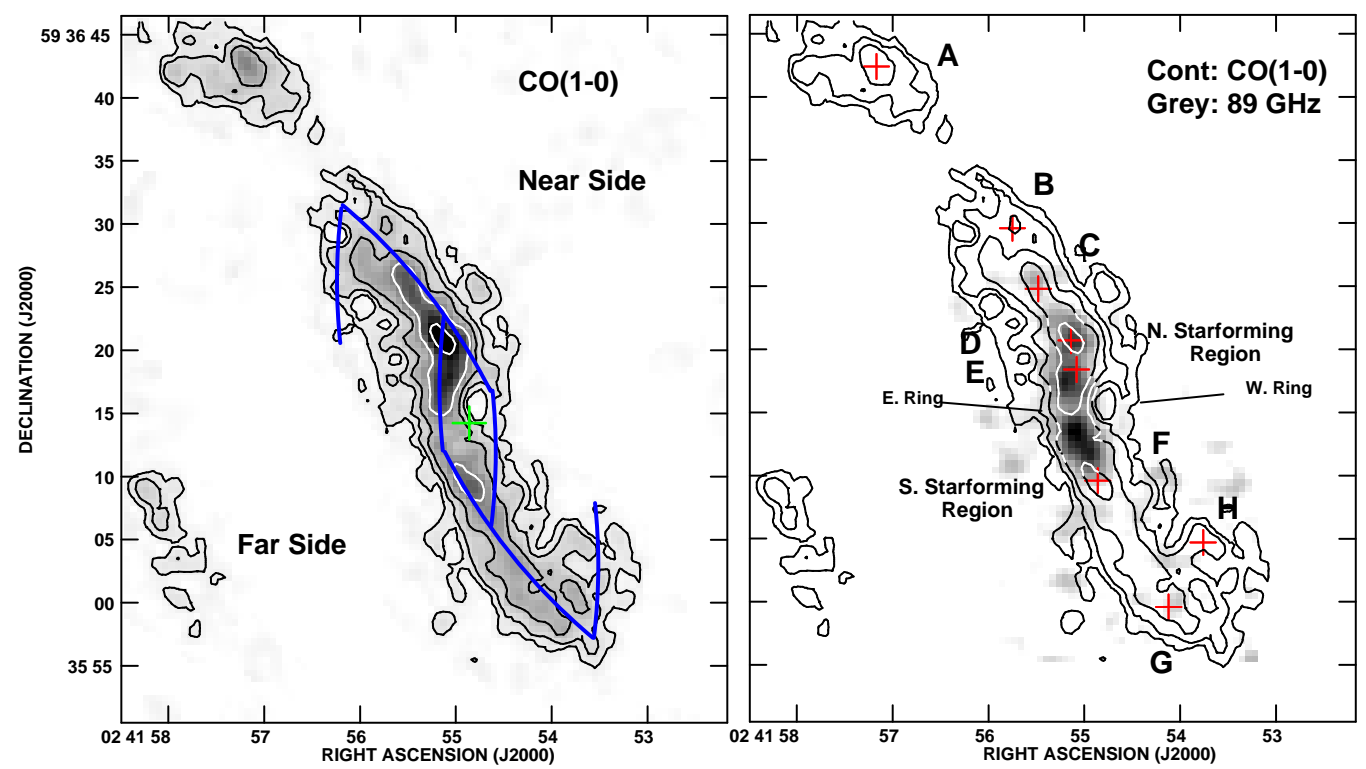

FIG. 1. - The basic structure of Maffei 2's nucleus. Left) The high resolution ${ }^{12} \mathrm{CO}(1-0)$ integrated intensity map from MTH08 with the location of the bar arms shown (in blue: online edition). Contours are $5.0 \mathrm{Jy} \mathrm{bm}^{-1} \mathrm{~km} \mathrm{~s}^{-1} \times 1,2,4,8,16$. A green cross marks the dynamical center. Right) The same CO contours overlaid on a greyscale of the $89 \mathrm{GHz}$ continuum (MTH08). The greyscale ranges from $0.5-5.0 \mathrm{mJy} \mathrm{bm}^{-1}$. The red crosses represent the location of the labeled GMCs. The location of the central ring and active star formation complexes are also noted.

TABLE 1

Molecule and Column Density Parameters

\begin{tabular}{|c|c|c|c|c|c|c|c|}
\hline $\begin{array}{l}\text { Molecular } \\
\text { Transition } \\
\end{array}$ & $\begin{array}{c}\mu \\
(D b y) \\
\end{array}$ & $\begin{array}{c}\mathrm{A} / \mathrm{B} / \mathrm{C} \\
(G H z) \\
\end{array}$ & $S_{u l} g_{K} g_{I}$ & $\begin{array}{l}E_{u} \\
(K)\end{array}$ & 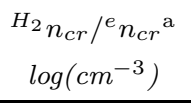 & $\begin{array}{l}N_{m o l} / I_{m o l} \\
\left(\frac{\mathrm{cm}^{2}}{K k m s^{-1}}\right)\end{array}$ & $\frac{N(50)}{N(10)} \mathrm{b}$ \\
\hline $\mathrm{SiO}(2-1 ; v=1)$ & 3.098 & $\cdots / 21.712 / \ldots$ & 2 & 1787 & $\cdots / \ldots$ & & \\
\hline $\mathrm{SiO}(2-1 ; v=0)$ & 3.098 & $\cdots / 21.712 / \ldots$ & 2 & 6.25 & $5.27 / 5.66$ & $1.86(12)$ & 2.94 \\
\hline $\mathrm{C}_{2} \mathrm{H}\left(1-0 ; \frac{3}{2}-\frac{1}{2}\right)$ & 0.8 & $\cdots / 43.675 / \quad \cdots$ & 1 & 4.19 & $5.13 / 6.24$ & $2.32(13)$ & 3.01 \\
\hline $\mathrm{C}_{2} \mathrm{H}\left(1-0 ; \frac{1}{2}-\frac{1}{2}\right)$ & 0.8 & $\cdots / 43.675 / \ldots$ & 1 & 4.19 & $5.13 / 6.24$ & $4.64(13)$ & 3.01 \\
\hline $\mathrm{HCO}+(1-0)$ & 3.888 & $\ldots / 44.594 / \ldots$ & 1 & 4.28 & $5.15 / 5.80$ & $9.51(11)$ & 3.36 \\
\hline $\mathrm{HNC}(1-0)$ & 3.05 & $\ldots / 45.332 / \ldots$ & 1 & 4.35 & $6.39 / 5.87$ & $1.51(12)$ & 2.94 \\
\hline $\mathrm{HC}_{3} \mathrm{~N}(10-9)$ & 3.72 & $\cdots / 4.5491 / \ldots$ & 10 & 24.02 & $5.86 / 6.87$ & $6.73(12)$ & 0.723 \\
\hline $\mathrm{N}_{2} \mathrm{H}^{+}(1-0)$ & 3.40 & $\cdots / 46.587 / \quad \cdots$ & 1 & 4.47 & $5.63 / 5.85$ & $1.16(12)$ & 3.00 \\
\hline $\mathrm{C}^{34} \mathrm{~S}(2-1)$ & 1.96 & $\ldots / 24.104 / \ldots$ & 2 & 6.94 & $5.65 / 6.17$ & $4.06(12)$ & 2.56 \\
\hline $\mathrm{CH}_{3} \mathrm{OH}\left(2_{k}-1_{k}\right)$ & 0.89 & $127.484 / 24.680 / 23.770$ & $4,3,4^{\mathrm{c}}$ & 6.98 & $4.83 / 6.39$ & $1.58(13)$ & 5.96 \\
\hline $\operatorname{HNCO}\left(5_{05}-4_{04}\right)$ & 1.60 & $912.711 / 11.071 / 10.911$ & 5 & 15.83 & $6.29 / 5.99$ & $9.27(12)$ & 3.15 \\
\hline
\end{tabular}

Note. - Data from Splatalogue, the JPL Molecular Spectroscopy Catalog (Pickett et al. 1998) and references within. Collisional coefficients are $\mathrm{C}^{34} \mathrm{~S}, \mathrm{HC}_{3} \mathrm{~N}$ (Green \& Chapman 1978), $\mathrm{N}_{2} \mathrm{H}^{+}$(Green 1975), $\mathrm{CH}_{3} \mathrm{OH}$ (Pottage et al. 2004), HNCO (Green 1986), HCO ${ }^{+}$(Flower 1999) and SiO (Davou \& Balanca 2006). For $\mathrm{HNC}$ and $\mathrm{C}_{2} \mathrm{H}$ we adopt the collision coefficients of HCN (Green \& Thaddeus 1974).

${ }^{\text {a }}$ The critical density of the transitions neglecting opacity effects $\left({ }^{H_{2}} n_{c r} \simeq \frac{A_{u l}}{C_{u l}}\right)$ and ${ }^{e} n_{c r}$ based on the formalism of Dickinson et al. (1977), with $\left[\mathrm{e}^{-}\right] \simeq 1.0 \times 10^{-5}$

b The ratio by which the derived column densities change when the $\mathrm{T}_{\mathrm{ex}}$ is changed from $10 \mathrm{~K} \rightarrow 50 \mathrm{~K}$.

${ }^{c} S_{u l} g_{K} g_{I}$ for the three blended $2_{1}-1_{1} \mathrm{E}, 2_{0}-1_{0} \mathrm{E}$, and $2_{0}-1_{0} \mathrm{~A}+$ transitions (eg., Turner 1991). 

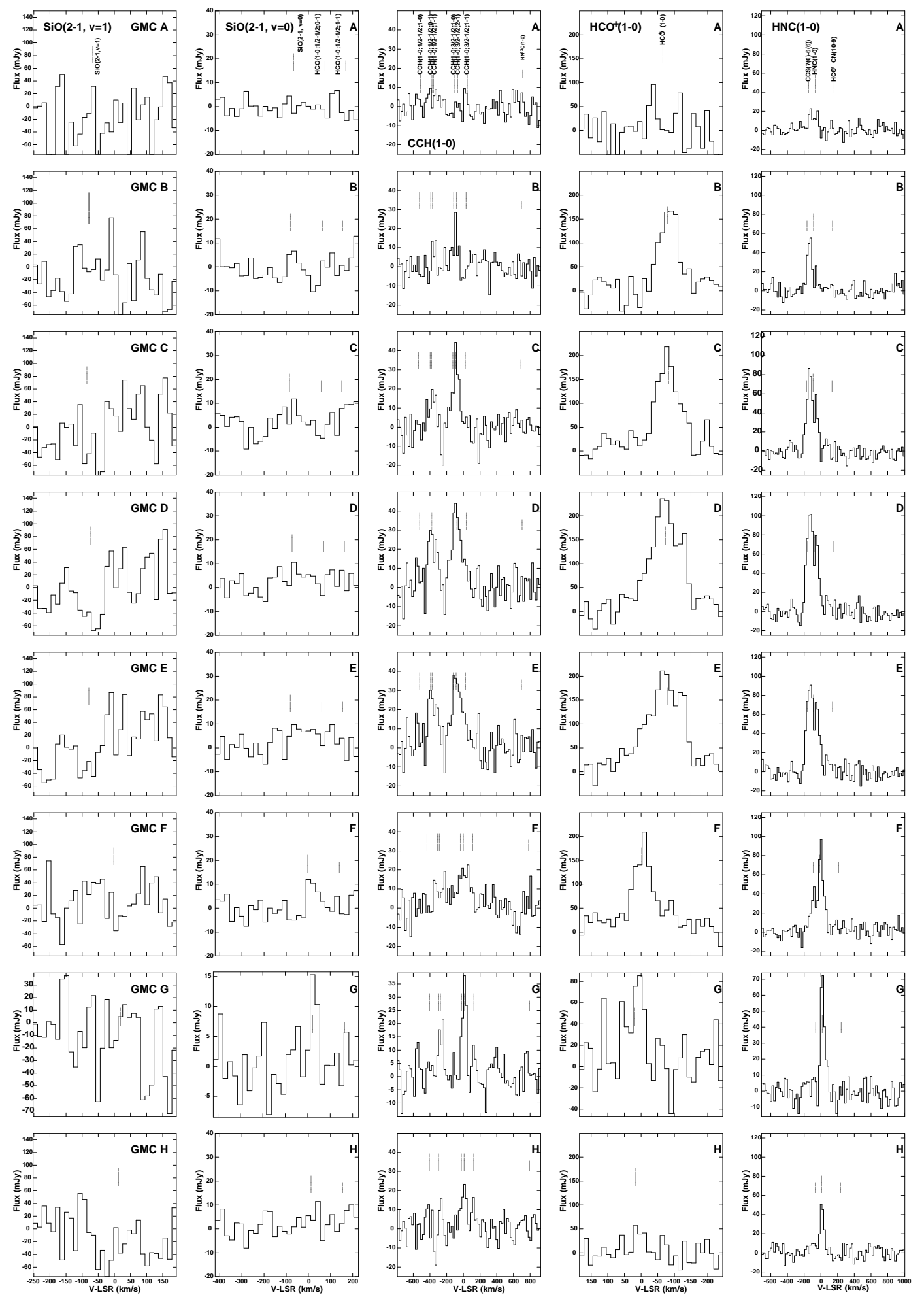

FIG. 2.- Spectra of the molecular transitions towards Maffei 2's GMCs. Spectra are summed over an 8 " aperture centered on the GMC positions indentified by MTH08. The vertical axis is the flux in the aperture and the $\mathrm{x}$ axis is LSR velocity. 

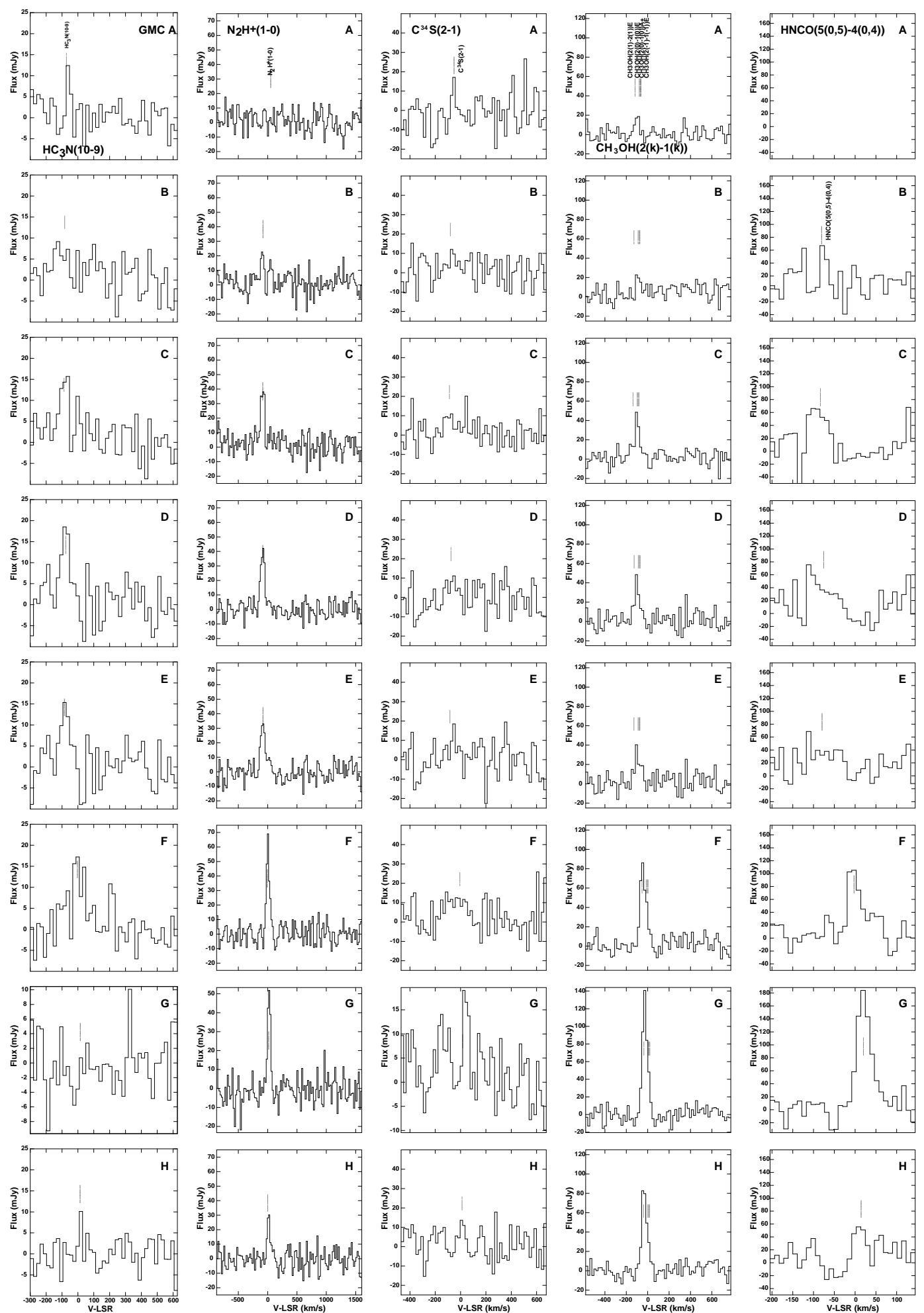

FIG. 2.- Spectra of the molecular transitions towards Maffei 2's GMCs, continued. 

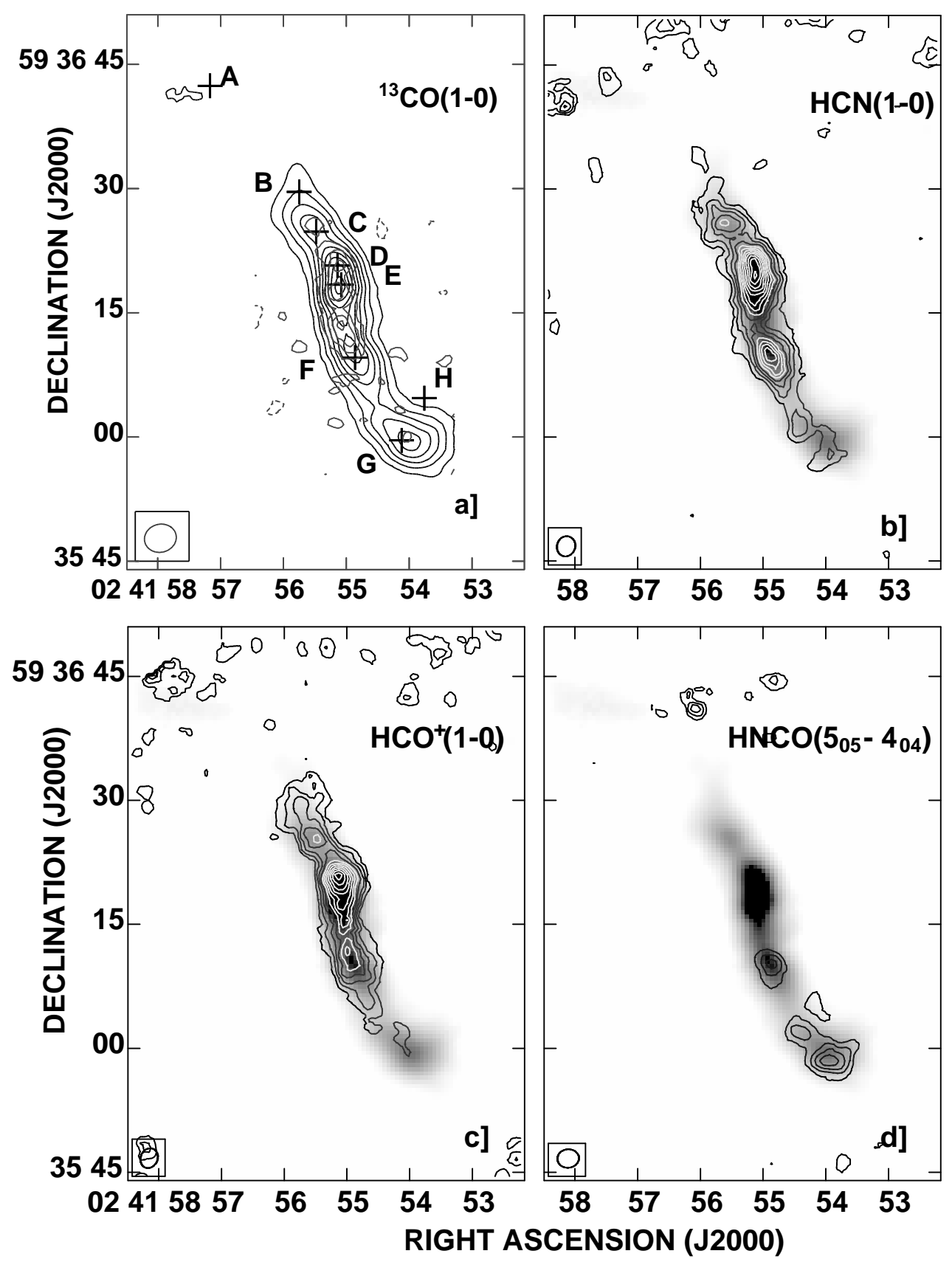

FIG. 3.- Integrated intensity maps of four molecular transitions in Maffei 2 observed with OVRO. a) The ${ }^{13} \mathrm{CO}(1-0)$ in steps of 0.75 Jy $\mathrm{bm}^{-1} \mathrm{~km} \mathrm{~s}^{-1}$. (MTH08) b) The HCN(1-0) (MTH08) in steps of $0.50 \mathrm{Jy} \mathrm{bm}^{-1} \mathrm{~km} \mathrm{~s}^{-1}$ with a resolution of $\left.2.5^{\prime \prime} \times 2.2^{\prime \prime} . c\right) \mathrm{The} \mathrm{HCO}^{+}(1-0)$ in steps of $0.50 \mathrm{Jy} \mathrm{bm}^{-1} \mathrm{~km} \mathrm{~s}^{-1}$ for a resolution given in Table 1 d) The $\mathrm{HNCO}\left(5_{05}-4_{04}\right)$ in steps of $0.40 \mathrm{Jy} \mathrm{bm}^{-1} \mathrm{~km} \mathrm{~s}^{-1}$. In all planes the greyscale is the ${ }^{13} \mathrm{CO}(1-0)$ integrated intensity. 

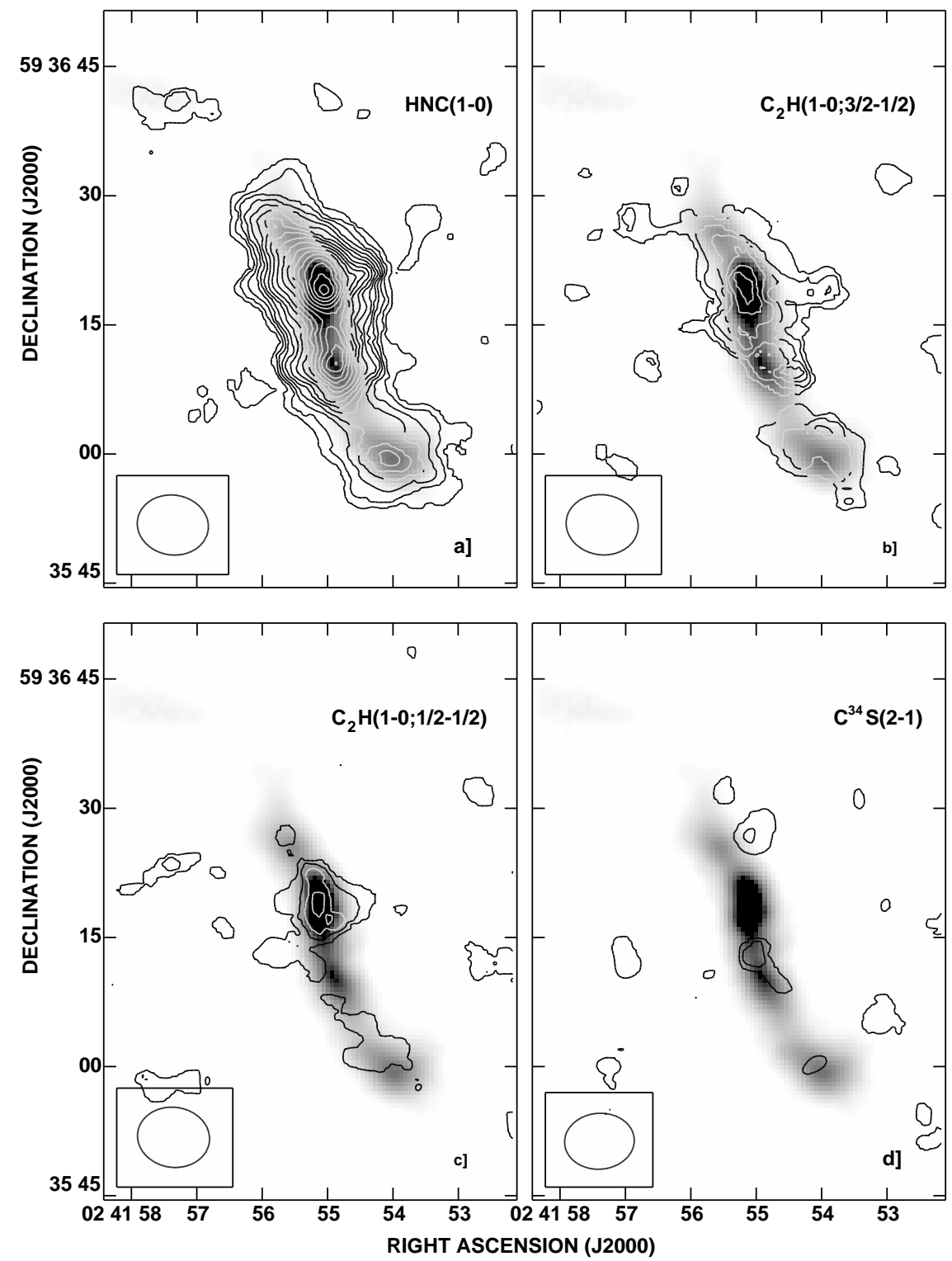

FIG. 4.- Integrated intensity maps of the molecular transitions in Maffei 2 observed with BIMA. a) The HNC(1-0) in steps of 1.0 Jy $\mathrm{bm}^{-1} \mathrm{~km} \mathrm{~s}^{-1}$. b) The $\mathrm{C}_{2} \mathrm{H}(1-0 ; 3 / 2-1 / 2)$ in steps of $1.1 \mathrm{Jy} \mathrm{bm}^{-1} \mathrm{~km} \mathrm{~s}^{-1}$. c) The $\mathrm{C}_{2} \mathrm{H}(1-0 ; 1 / 2-1 / 2)$ in steps of $1.1 \mathrm{Jy} \mathrm{bm}^{-1} \mathrm{~km} \mathrm{~s}^{-1}$. d) The $\mathrm{C}^{34} \mathrm{~S}(2-1)$ in steps of $1.2 \mathrm{Jy} \mathrm{bm}^{-1} \mathrm{~km} \mathrm{~s}^{-1}$. In all planes the greyscale is the ${ }^{13} \mathrm{CO}(1-0)$ integrated intensity. 

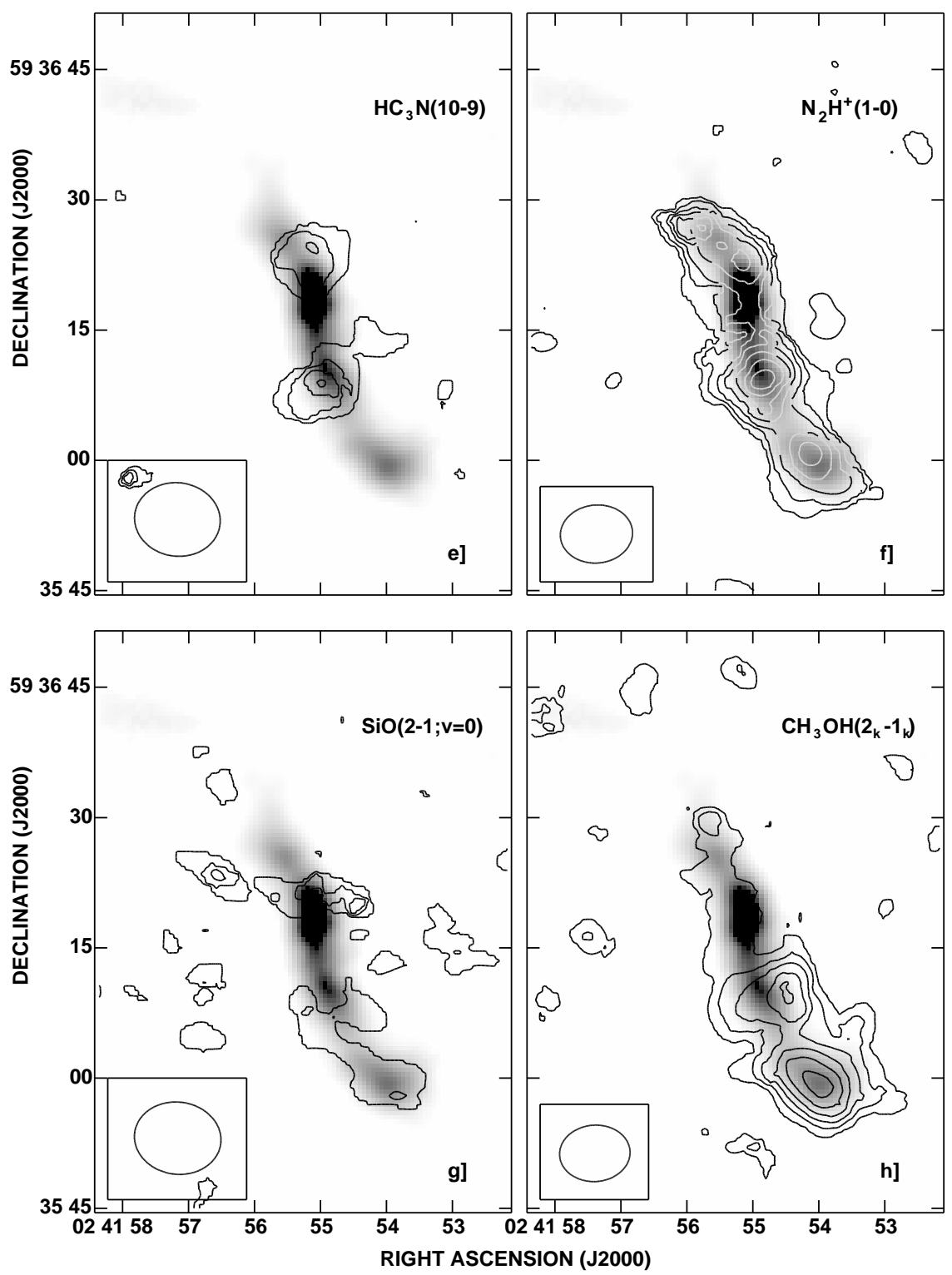

FIG. 4.- Integrated intensity maps of the molecular transitions in Maffei 2 observed with BIMA, continued. e) The HC 3 N(10-9) integrated intensity in steps of levels $0.60 \mathrm{Jy} \mathrm{bm}^{-1} \mathrm{~km} \mathrm{~s}^{-1}$. f) The $\mathrm{N}_{2} \mathrm{H}^{+}(1-0)$ in steps of $\left.0.75 \mathrm{Jy} \mathrm{bm}^{-1} \mathrm{~km} \mathrm{~s}^{-1} . g\right) \mathrm{The} \mathrm{SiO}(2-1 ; \mathrm{v}=0)$ in steps of $0.40 \mathrm{Jy} \mathrm{bm}^{-1} \mathrm{~km} \mathrm{~s}^{-1}$. h) The $\mathrm{CH}_{3} \mathrm{OH}\left(2_{k}-1_{k}\right)$ in steps of $1.5 \mathrm{Jy} \mathrm{bm}^{-1} \mathrm{~km} \mathrm{~s}^{-1}$. In all planes the greyscale is the ${ }^{13} \mathrm{CO}(1-0)$ integrated intensity. 


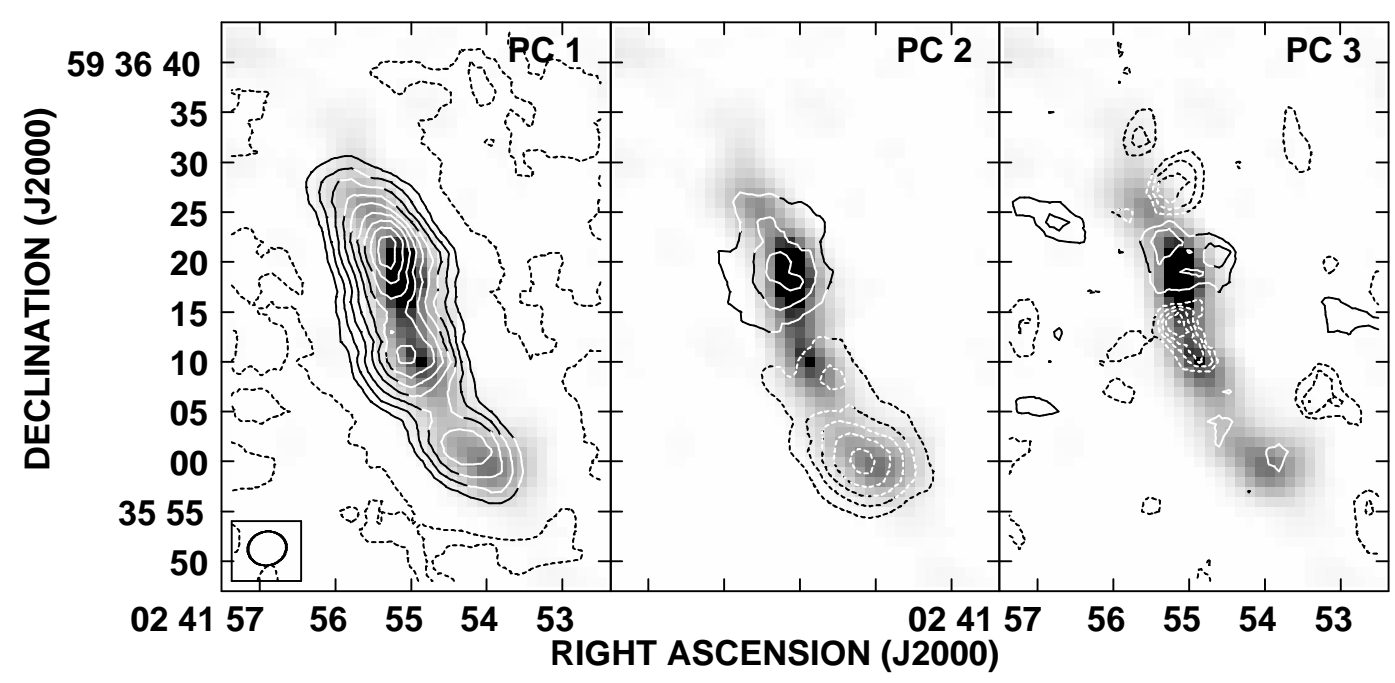

FIG. 5.- Maps of the first three principal components of the molecular distribution.

TABLE 2

Observational Data

\begin{tabular}{|c|c|c|c|c|c|c|c|c|}
\hline Transition & $\begin{array}{l}\text { Dates } \\
(M M Y Y)\end{array}$ & Array & $\begin{array}{c}\text { Frequency } \\
\quad(\mathrm{GHz})\end{array}$ & $\begin{array}{l}\mathrm{T}_{\text {sys }} \\
(K)\end{array}$ & $\begin{array}{c}\Delta V_{\text {chan }} \\
\left(k m s^{-1}\right)\end{array}$ & $\begin{array}{c}\text { Beam } \\
\left({ }^{\prime \prime} x^{\prime \prime}\right)\end{array}$ & $\mathrm{K} / \mathrm{Jy}$ & $\begin{array}{c}\text { Noise } \\
\left(m J y b m^{-1}\right)\end{array}$ \\
\hline \multicolumn{9}{|l|}{ BIMA: } \\
\hline $\mathrm{SiO}(2-1 ; \mathrm{v}=0)^{\mathrm{a}}$ & 0902-0303 & $\mathrm{B}, \mathrm{C}, \mathrm{D}$ & 86.847 & $140-1000$ & 21.57 & $10.0 \times 8.3 ; 83$ & 1.68 & 10 \\
\hline $\mathrm{C}_{2} \mathrm{H}(1-0 ; 3 / 2-1 / 2)^{\mathrm{a}}$ & 0902-0303 & B,C,D & 87.317 & $140-1000$ & 21.46 & $8.4 \times 7.0 ;-84$ & 2.75 & 11 \\
\hline $\operatorname{HNC}(1-0)^{a}$ & 0902-0303 & B,C,D & 90.664 & $140-1000$ & 20.67 & $8.3 \times 7.0 ; 81$ & 2.59 & 11 \\
\hline $\mathrm{HC}_{3} \mathrm{~N}(10-9)^{\mathrm{a}}$ & 0902-0303 & $\mathrm{B}, \mathrm{C}, \mathrm{D}$ & 90.979 & $140-1000$ & 20.60 & $9.9 \times 8.5 ; 83$ & 1.77 & 7.2 \\
\hline $\mathrm{N}_{2} \mathrm{H}^{+}(1-0)^{\mathrm{a}}$ & 0902-0303 & $\mathrm{B}, \mathrm{C}, \mathrm{D}$ & 93.174 & $130-1100$ & 20.12 & $8.3 \times 6.7 ;-85$ & 2.53 & 9.0 \\
\hline $\mathrm{C}^{34} \mathrm{~S}(2-1)^{\mathrm{a}}$ & 0902-0303 & $\mathrm{B}, \mathrm{C}, \mathrm{D}$ & 96.413 & $130-1100$ & 19.43 & $8.1 \times 6.5 ;-86$ & 2.51 & 11 \\
\hline $\begin{array}{l}\mathrm{CH}_{3} \mathrm{OH}\left(2_{k}-1_{k}\right)^{\mathrm{a}} \\
\text { OVRO: }\end{array}$ & 0902-0303 & $\mathrm{B}, \mathrm{C}, \mathrm{D}$ & 96.741 & $130-1100$ & 19.37 & $8.1 \times 6.5 ;-86$ & 2.48 & 15 \\
\hline $\mathrm{SiO}(2-1 ; \mathrm{v}=1)^{\mathrm{b}}$ & 0199-0399 & $\mathrm{L}, \mathrm{H}, \mathrm{UH}$ & 86.243 & $300-410$ & 13.90 & $2.3 \times 2.0 ;-56$ & 36.5 & 9.5 \\
\hline $\mathrm{HCO}^{+}(1-0)^{\mathrm{b}}$ & 0199-0399 & $\mathrm{L}, \mathrm{H}, \mathrm{UH}$ & 89.189 & $300-410$ & 13.44 & $2.5 \times 2.1 ;-24$ & 30.5 & 6.7 \\
\hline $\operatorname{HNCO}\left(5_{05}-4_{04}\right)^{\mathrm{c}}$ & 1098-0199 & $\mathrm{L}, \mathrm{H}$ & 109.906 & $240-430$ & 10.92 & $2.6 \times 2.2 ;-84$ & 17.3 & 6.3 \\
\hline
\end{tabular}

a Phase Center \#1: $\alpha=02^{h} 41^{m} 55^{s} .0 \quad \delta=+59^{\circ} 36^{\prime} 15$. $^{\prime \prime} 0(\mathrm{~J} 2000) ; \mathrm{v}_{l s r}=-15 \mathrm{~km} \mathrm{~s}^{-1}$

b Phase Center \#1: $\alpha=02^{h} 38^{m} 08^{s} .0 \quad \delta=+59^{\circ} 23^{\prime} 20 .^{\prime \prime} 0$ (B1950); $\mathrm{v}_{l s r}=-30 \mathrm{~km} \mathrm{~s}^{-1}$

Phase Center \#2: $\alpha=02^{h} 38^{m} 08^{s} .25 \quad \delta=+59^{\circ} 23^{\prime} 27$. $^{\prime \prime} 0$ (B1950); $\mathrm{v}_{l s r}=-30 \mathrm{~km} \mathrm{~s}^{-1}$

${ }^{c}$ Phase Center \#1: $\alpha=02^{h} 38^{m} 08^{s} .0 \quad \delta=+59^{\circ} 23^{\prime} 20{ }^{\prime \prime} 0$ (B1950); $\mathrm{v}_{l s r}=-30 \mathrm{~km} \mathrm{~s}^{-1}$

Phase Center \#2: $\alpha=02^{h} 38^{m} 08^{s} .25 \quad \delta=+59^{\circ} 23^{\prime} 2$. $^{\prime \prime} 0$ (B1950); $\mathrm{v}_{l s r}=-30 \mathrm{~km} \mathrm{~s}^{-1}$ 
TABLE 3

Measured Intensities, Linewidths \& Centroids

\begin{tabular}{|c|c|c|c|c|c|}
\hline $\begin{array}{l}\mathrm{GMC} \\
\alpha_{o}, \delta_{o}\end{array}$ & Transition & $\begin{array}{c}\mathrm{I}_{m o l} \\
\left(K k m s^{-1}\right)\end{array}$ & $\begin{array}{c}\mathrm{T}_{b} \\
(m K)\end{array}$ & $\begin{array}{c}v_{o} \\
\left(k m s^{-1}\right)\end{array}$ & $\begin{array}{c}\Delta v \\
\left(k m s^{-1}\right)\end{array}$ \\
\hline $\mathrm{A}$ & $\mathrm{SiO}(1-0 ; \mathrm{v}=1)$ & $<19$ & $<88$ & $\ldots$ & $\ldots$ \\
\hline$(02: 41: 57.17)$ & $\mathrm{SiO}(1-0 ; \mathrm{v}=0)$ & $<1.0$ & $<23$ & $\cdots$ & $\cdots$ \\
\hline \multirow[t]{9}{*}{$(59: 36: 42.4)$} & $\mathrm{C}_{2} \mathrm{H}\left(\frac{3}{2}-\frac{1}{2}\right)$ & $<3.3$ & $<34$ & $\cdots$ & $\cdots$ \\
\hline & $\mathrm{C}_{2} \mathrm{H}\left(\frac{1}{2}-\frac{1}{2}\right)$ & $<3.3$ & $<34$ & $\ldots$ & $\ldots$ \\
\hline & $\mathrm{HCO}^{+}(1-0)$ & $16 \pm 7$ & $270 \pm 38$ & $-34 \pm 5$ & $21 \pm 10$ \\
\hline & $\mathrm{HNC}(1-0)$ & $<2.9$ & $45 \pm 16$ & $-100 \pm 11$ & $120 \pm 25$ \\
\hline & $\mathrm{HC}_{3} \mathrm{~N}(10-9)$ & $<1.0$ & $<26$ & $\ldots$ & $\ldots$ \\
\hline & $\mathrm{N}_{2} \mathrm{H}^{+}(1-0)$ & $<1.9$ & $<30$ & $\cdots$ & $\ldots$ \\
\hline & $\mathrm{C}^{34} \mathrm{~S}(2-1)$ & $<2.2$ & $<28$ & $\ldots$ & $\ldots$ \\
\hline & $\mathrm{CH}_{3} \mathrm{OH}\left(2_{k}-1_{k}\right)$ & $<3.2$ & $46 \pm 19$ & $-100 \pm 6$ & $45 \pm 15$ \\
\hline & $\operatorname{HNCO}\left(5_{05}-4_{04}\right)$ & $\ldots$ & $\ldots$ & $\ldots$ & $\ldots$ \\
\hline B & $\mathrm{SiO}(1-0 ; \mathrm{v}=1)$ & $<19$ & $<88$ & $\ldots$ & $\ldots$ \\
\hline$(02: 41: 55.75)$ & $\mathrm{SiO}(1-0 ; \mathrm{v}=0)$ & $<1.0$ & $35 \pm 12$ & $-67 \pm 13$ & $55 \pm 30$ \\
\hline \multirow[t]{9}{*}{$(59: 36: 29.6)$} & $\mathrm{C}_{2} \mathrm{H}\left(\frac{3}{2}-\frac{1}{2}\right)$ & $<3.3$ & $<34$ & $\ldots$ & $\ldots$ \\
\hline & $\mathrm{C}_{2} \mathrm{H}\left(\frac{1}{2}-\frac{1}{2}\right)$ & $<3.3$ & $<34$ & $\cdots$ & $\cdots$ \\
\hline & $\mathrm{HCO}^{+}(1-0)$ & $61 \pm 7$ & $470 \pm 38$ & $-84 \pm 3$ & $68 \pm 7$ \\
\hline & $\mathrm{HNC}(1-0)$ & $9.1 \pm 1$ & $150 \pm 16$ & $-134 \pm 3$ & $65 \pm 7$ \\
\hline & $\mathrm{HC}_{3} \mathrm{~N}(10-9)$ & $<1.0$ & $<26$ & $\ldots$ & $\ldots$ \\
\hline & $\mathrm{N}_{2} \mathrm{H}^{+}(1-0)$ & $5.1 \pm 1$ & $60 \pm 15$ & $-98 \pm 6$ & $50 \pm 10$ \\
\hline & $\mathrm{CH}_{3} \mathrm{OH}\left(2_{k}-1_{k}\right)$ & $10 \pm 2$ & $53 \pm 19$ & $-87 \pm 8$ & $48 \pm 20$ \\
\hline & $\mathrm{C}^{34} \mathrm{~S}(2-1)$ & $<2.2$ & $<28$ & $\ldots$ & $\ldots$ \\
\hline & $\operatorname{HNCO}\left(5_{05}-4_{04}\right)$ & $\lesssim 4.8$ & $50 \pm 29$ & $-112 \pm 20$ & $110 \pm 50$ \\
\hline $\mathrm{C}$ & $\mathrm{SiO}(1-0 ; \mathrm{v}=1)$ & $<19$ & $<88$ & $\ldots$ & $\ldots$ \\
\hline$(02: 41: 55.48)$ & $\mathrm{SiO}(1-0 ; \mathrm{v}=0)$ & $<1.0$ & $24 \pm 12$ & $-80 \pm 20$ & $83 \pm 47$ \\
\hline \multirow[t]{9}{*}{$(59: 36: 24.8)$} & $\mathrm{C}_{2} \mathrm{H}\left(\frac{3}{2}-\frac{1}{2}\right)$ & $11 \pm 2$ & $100 \pm 17$ & $-88 \pm 6$ & $93 \pm 15$ \\
\hline & $\mathrm{C}_{2} \mathrm{H}\left(\frac{1}{2}-\frac{1}{2}\right)$ & $3.9 \pm 2$ & $48 \pm 17$ & $-76 \pm 17$ & $90 \pm 40$ \\
\hline & $\mathrm{HCO}^{+}(1-0)$ & $82 \pm 7$ & $520 \pm 38$ & $-78 \pm 3$ & $83 \pm 6$ \\
\hline & $\mathrm{HNC}(1-0)$ & $24 \pm 1$ & $200 \pm 16$ & $-120 \pm 4$ & $120 \pm 8$ \\
\hline & $\mathrm{HC}_{3} \mathrm{~N}(10-9)$ & $2.8 \pm 0.5$ & $42 \pm 13$ & $-88 \pm 7$ & $65 \pm 16$ \\
\hline & $\mathrm{N}_{2} \mathrm{H}^{+}(1-0)$ & $9.6 \pm 1$ & $110 \pm 15$ & $-88 \pm 5$ & $77 \pm 10$ \\
\hline & $\mathrm{CH}_{3} \mathrm{OH}\left(2_{k}-1_{k}\right)$ & $5.0 \pm 2$ & $100 \pm 19$ & $-99 \pm 4$ & $48 \pm 8$ \\
\hline & $\mathrm{C}^{34} \mathrm{~S}(2-1)$ & $<2.2$ & $<28$ & $\ldots$ & $\ldots$ \\
\hline & $\operatorname{HNCO}\left(5_{05}-4_{04}\right)$ & $\lesssim 4.8$ & $130 \pm 29$ & $-89 \pm 5$ & $42 \pm 10$ \\
\hline $\mathrm{D}$ & $\mathrm{SiO}(1-0 ; \mathrm{v}=1)$ & $<19$ & $<88$ & $\ldots$ & $\ldots$ \\
\hline$(02: 41: 55.14)$ & $\mathrm{SiO}(1-0 ; \mathrm{v}=0)$ & $2.5 \pm 0.5$ & $<23$ & $\cdots$ & $\cdots$ \\
\hline \multirow[t]{9}{*}{$(59: 36: 20.7)$} & $\mathrm{C}_{2} \mathrm{H}\left(\frac{3}{2}-\frac{1}{2}\right)$ & $17 \pm 2$ & $110 \pm 17$ & $-82 \pm 9$ & $130 \pm 22$ \\
\hline & $\mathrm{C}_{2} \mathrm{H}\left(\frac{1}{2}-\frac{1}{2}\right)$ & $11 \pm 2$ & $80 \pm 17$ & $-77 \pm 17$ & $120 \pm 35$ \\
\hline & $\mathrm{HCO}^{+}(1-0)$ & $180 \pm 7$ & $580 \pm 38$ & $-74 \pm 3$ & $110 \pm 7$ \\
\hline & $\mathrm{HNC}(1-0)$ & $41 \pm 1$ & $250 \pm 16$ & $-109 \pm 4$ & $140 \pm 8$ \\
\hline & $\mathrm{HC}_{3} \mathrm{~N}(10-9)$ & $3.2 \pm 0.5$ & $50 \pm 13$ & $-80 \pm 6$ & $67 \pm 15$ \\
\hline & $\mathrm{N}_{2} \mathrm{H}^{+}(1-0)$ & $9.4 \pm 1$ & $110 \pm 15$ & $-87 \pm 4$ & $82 \pm 8$ \\
\hline & $\mathrm{CH}_{3} \mathrm{OH}\left(2_{k}-1_{k}\right)$ & $5.5 \pm 2$ & $95 \pm 19$ & $-101 \pm 5$ & $57 \pm 12$ \\
\hline & $\mathrm{C}^{34} \mathrm{~S}(2-1)$ & $<2.2$ & $<28$ & $\ldots$ & $\ldots$ \\
\hline & $\mathrm{HNCO}\left(5_{05}-4_{04}\right)$ & $\lesssim 4.8$ & $86 \pm 29$ & $-90 \pm 11$ & $82 \pm 15$ \\
\hline $\mathrm{E}$ & $\mathrm{SiO}(1-0 ; \mathrm{v}=1)$ & $<19$ & $<88$ & $\ldots$ & $\ldots$ \\
\hline$(02: 41: 55.08)$ & $\mathrm{SiO}(1-0 ; \mathrm{v}=0)$ & $\sim 0.77$ & $22 \pm 12$ & $-2.0 \pm 26$ & $220 \pm 60$ \\
\hline \multirow[t]{9}{*}{$(59: 36: 18.4)$} & $\mathrm{C}_{2} \mathrm{H}\left(\frac{3}{2}-\frac{1}{2}\right)$ & $19 \pm 2$ & $97 \pm 17$ & $-73 \pm 12$ & $160 \pm 28$ \\
\hline & $\mathrm{C}_{2} \mathrm{H}\left(\frac{1}{2}-\frac{1}{2}\right)$ & $13 \pm 2$ & $77 \pm 17$ & $-72 \pm 16$ & $130 \pm 38$ \\
\hline & $\mathrm{HCO}^{+}(1-0)$ & $130 \pm 7$ & $520 \pm 38$ & $-73 \pm 4$ & $130 \pm 8$ \\
\hline & $\mathrm{HNC}(1-0)$ & $44 \pm 1$ & $220 \pm 16$ & $-104 \pm 4$ & $150 \pm 10$ \\
\hline & $\mathrm{HC}_{3} \mathrm{~N}(10-9)$ & $2.1 \pm 0.5$ & $39 \pm 13$ & $-80 \pm 8$ & $67 \pm 18$ \\
\hline & $\mathrm{N}_{2} \mathrm{H}^{+}(1-0)$ & $6.6 \pm 1$ & $85 \pm 15$ & $-83 \pm 5$ & $92 \pm 12$ \\
\hline & $\mathrm{CH}_{3} \mathrm{OH}\left(2_{k}-1_{k}\right)$ & $6.7 \pm 2$ & $70 \pm 19$ & $-94 \pm 8$ & $75 \pm 18$ \\
\hline & $\mathrm{C}^{34} \mathrm{~S}(2-1)$ & $<2.2$ & $<28$ & $\ldots$ & $\cdots$ \\
\hline & $\mathrm{HNCO}\left(5_{05}-4_{04}\right)$ & $<4.8$ & $<58$ & $\cdots$ & $\cdots$ \\
\hline $\mathrm{F}$ & $\mathrm{SiO}(1-0 ; \mathrm{v}=1)$ & $<19$ & $<88$ & $\ldots$ & $\ldots$ \\
\hline$(02: 41: 54.86)$ & $\mathrm{SiO}(1-0 ; \mathrm{v}=0)$ & $<1.0$ & $35 \pm 12$ & $17 \pm 8$ & $50 \pm 18$ \\
\hline \multirow[t]{9}{*}{$(59: 36: 09.6)$} & $\mathrm{C}_{2} \mathrm{H}\left(\frac{3}{2}-\frac{1}{2}\right)$ & $9.4 \pm 2$ & $63 \pm 17$ & $10 \pm 12$ & $130 \pm 50$ \\
\hline & $\mathrm{C}_{2} \mathrm{H}\left(\frac{1}{2}-\frac{1}{2}\right)$ & $<3.3$ & $\sim 46$ & $\cdots$ & $\cdots$ \\
\hline & $\mathrm{HCO}^{+}(1-0)$ & $85 \pm 7$ & $450 \pm 38$ & $-6.9 \pm 4$ & $83 \pm 8$ \\
\hline & $\mathrm{HNC}(1-0)$ & $36 \pm 1$ & $250 \pm 16$ & $-0.9 \pm 3$ & $67 \pm 17$ \\
\hline & $\mathrm{HC}_{3} \mathrm{~N}(10-9)$ & $4.1 \pm 0.5$ & $31 \pm 13$ & $0.1 \pm 13$ & $130 \pm 30$ \\
\hline & $\mathrm{N}_{2} \mathrm{H}^{+}(1-0)$ & $15 \pm 1$ & $150 \pm 15$ & $5.4 \pm 6$ & $70 \pm 10$ \\
\hline & $\mathrm{CH}_{3} \mathrm{OH}\left(2_{k}-1_{k}\right)$ & $15 \pm 2$ & $190 \pm 19$ & $-43 \pm 3$ & $68 \pm 5$ \\
\hline & $\mathrm{C}^{34} \mathrm{~S}(2-1)$ & $5.0 \pm 1$ & $\sim 30$ & $-46 \pm 26$ & $180 \pm 62$ \\
\hline & $\operatorname{HNCO}\left(5_{05}-4_{04}\right)$ & $22 \pm 2$ & $190 \pm 29$ & $-1.6 \pm 3$ & $40 \pm 7$ \\
\hline
\end{tabular}


TABLE 3

Measured Intensities, Linewidths \& Centroids

\begin{tabular}{|c|c|c|c|c|c|}
\hline $\begin{array}{l}\mathrm{GMC} \\
\alpha_{o}, \delta_{o}\end{array}$ & Transition & $\begin{array}{c}\mathrm{I}_{m o l} \\
\left(K \mathrm{~km} \mathrm{~s}^{-1}\right)\end{array}$ & $\begin{array}{c}\mathrm{T}_{b} \\
(m K)\end{array}$ & $\begin{array}{c}v_{o} \\
\left(k m s^{-1}\right)\end{array}$ & $\begin{array}{c}\Delta v \\
\left(k m s^{-1}\right)\end{array}$ \\
\hline $\begin{array}{l}\mathrm{G} \\
(02: 41: 54.12) \\
(59: 35: 59.6)\end{array}$ & $\begin{array}{c}\mathrm{SiO}(1-0 ; \mathrm{v}=1) \\
\mathrm{SiO}(1-0 ; \mathrm{v}=0) \\
\mathrm{C}_{2} \mathrm{H}\left(\frac{3}{2}-\frac{1}{2}\right) \\
\mathrm{C}_{2} \mathrm{H}\left(\frac{1}{2}-\frac{1}{2}\right) \\
\mathrm{HCO}^{+}(1-0) \\
\mathrm{HNC}(1-0) \\
\mathrm{HC}_{3} \mathrm{~N}(10-9) \\
\mathrm{N}_{2} \mathrm{H}^{+}(1-0) \\
\mathrm{CH}_{3} \mathrm{OH}\left(2_{k}-1_{k}\right) \\
\mathrm{C}^{34} \mathrm{~S}(2-1) \\
\mathrm{HNCO}(505-404) \\
\mathrm{SiO}(1-0 ; \mathrm{v}=1) \\
\mathrm{SiO}(1-0 ; \mathrm{v}=0) \\
\mathrm{C}_{2} \mathrm{H}\left(\frac{3}{2}-\frac{1}{2}\right) \\
\mathrm{C}_{2} \mathrm{H}\left(\frac{1}{2}-\frac{1}{2}\right) \\
\mathrm{HCO}(1-0) \\
\mathrm{HNC}(1-0) \\
\mathrm{HC}_{3} \mathrm{~N}(10-9) \\
\mathrm{N}_{2} \mathrm{H}^{+}(1-0) \\
\mathrm{CH}_{3} \mathrm{OH}\left(2_{k}-1_{k}\right) \\
\mathrm{C}^{34} \mathrm{~S}(2-1) \\
\mathrm{HNC}^{2}\left(5_{05}-4_{04}\right)\end{array}$ & $\begin{array}{c}<19 \\
1.2 \pm 0.5 \\
6.1 \pm 2 \\
4.1 \pm 2 \\
\sim 15 \pm 7 \\
17 \pm 1 \\
<1.5 \\
10 \pm 1 \\
25 \pm 2 \\
3.3 \pm 2 \\
33 \pm 2 \\
<19 \\
<1.0 \\
<3.3 \\
<3.3 \\
<15 \\
7.8 \pm 1 \\
<1.0 \\
<1.9 \\
8.4 \pm 2 \\
<2.2 \\
\lesssim 4.8\end{array}$ & $\begin{array}{c}<88 \\
52 \pm 17 \\
110 \pm 17 \\
51 \pm 17 \\
200 \pm 38 \\
200 \pm 16 \\
<26 \\
140 \pm 15 \\
300 \pm 19 \\
47 \pm 19 \\
320 \pm 29 \\
<88 \\
<23 \\
60 \pm 17 \\
<34 \\
150 \pm 38 \\
130 \pm 16 \\
<26 \\
73 \pm 15 \\
200 \pm 19 \\
<28 \\
110 \pm 29\end{array}$ & $\begin{array}{c}\cdots \\
28 \pm 4 \\
12 \pm 4 \\
27 \pm 12 \\
15 \pm 7 \\
20 \pm 2 \\
\cdots \\
23 \pm 3 \\
-27 \pm 2 \\
40 \pm 6 \\
21 \pm 2 \\
\cdots \\
\cdots \\
5.8 \pm 9 \\
\cdots \\
6.2 \pm 6 \\
10 \pm 5 \\
\cdots \\
20 \pm 6 \\
-32 \pm 2 \\
\cdots \\
11 \pm 4\end{array}$ & $\begin{array}{c}\cdots \\
32 \pm 13 \\
55 \pm 10 \\
72 \pm 28 \\
62 \pm 15 \\
55 \pm 5 \\
\cdots \\
57 \pm 7 \\
55 \pm 3 \\
50 \pm 15 \\
43 \pm 3 \\
\cdots \\
\cdots \\
78 \pm 22 \\
\cdots \\
35 \pm 13 \\
58 \pm 17 \\
\cdots \\
57 \pm 10 \\
60 \pm 5 \\
\cdots \\
32 \pm 10\end{array}$ \\
\hline
\end{tabular}

Note. - $\mathrm{T}_{b}$ is the main-beam brightness temperature based on a $8^{\prime \prime}$ aperture. $I_{m o l}$ is the peak integrated intensity in units of $\mathrm{K}$ km $\mathrm{s}^{-1}$ for the resolution given in Table 1 Uncertainties are based on the RMS noise for the temperatures and intensities, and $1 \sigma$ from the least-squared gaussian fits for the velocity information. Upper limits represent $2 \sigma$ values.
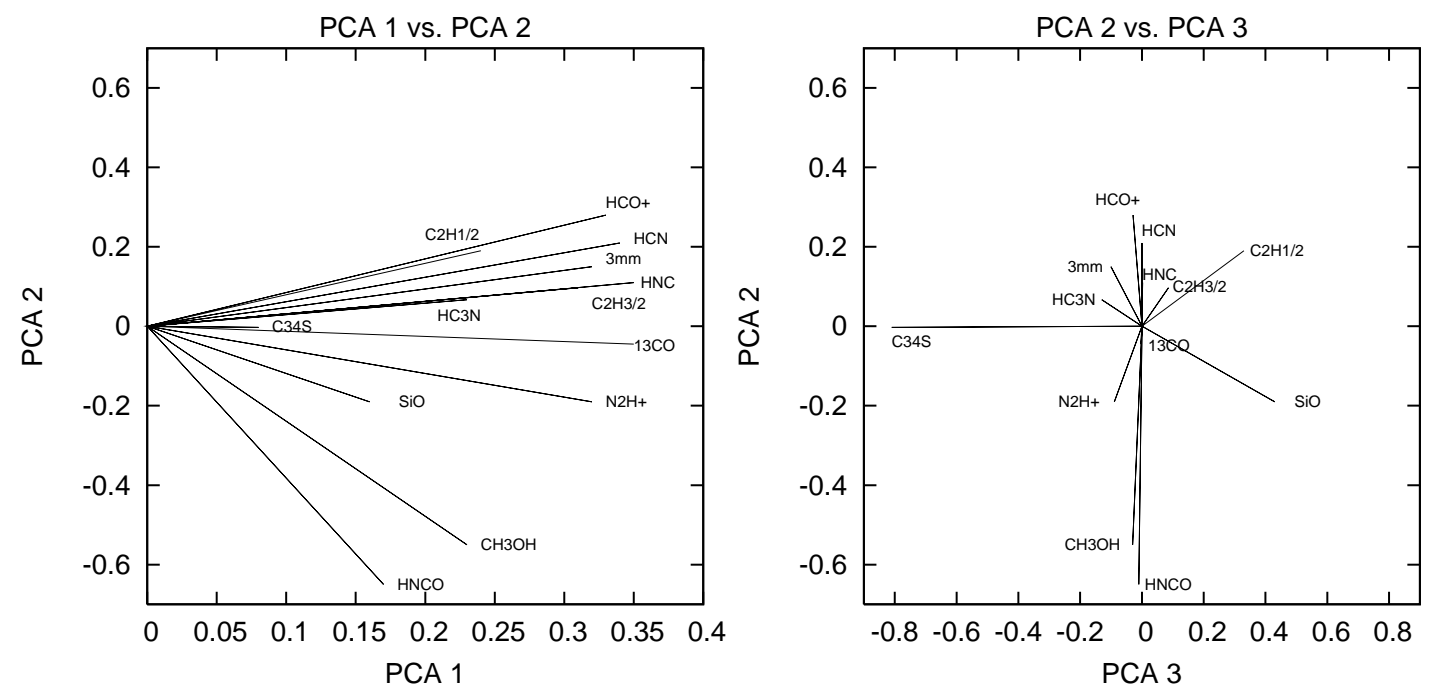

FIG. 6. - Projections of each map onto the first three principal component maps. Left) Projections onto the first and second principal components. Right) Projections onto the second and third component, while the figure plane geometry is such that it may be seen as a view looking to the left down the PC 1 axis. 

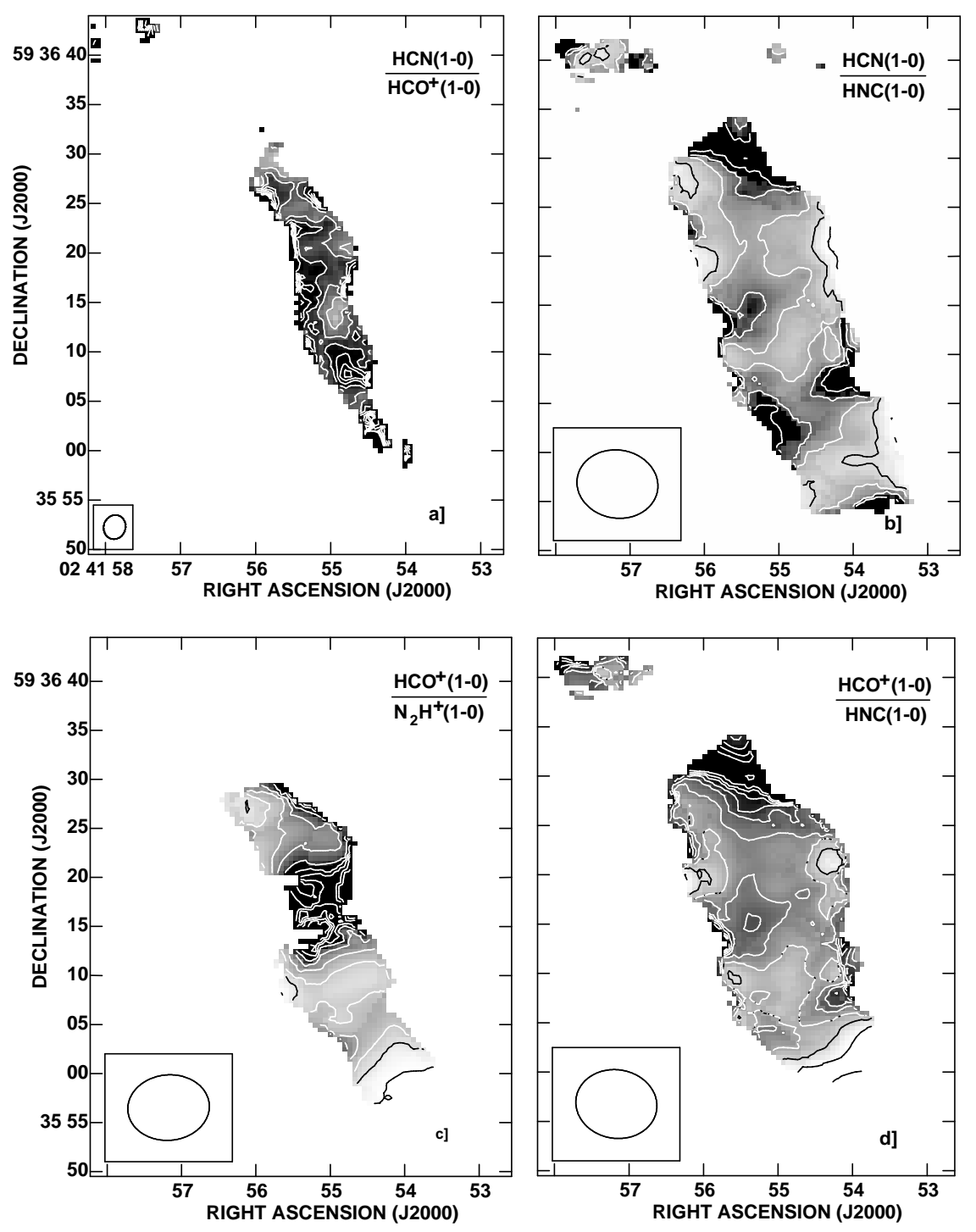

Fig. 7. - a) The $\mathrm{HCN}(1-0) / \mathrm{HCO}^{+}(1-0)$ line ratio. The greyscale ranges from 0 to 1.5 with dark being large ratios and contours are in steps of 0.333 for the resolution of the $\operatorname{HNC}(1-0)$ dataset. $\mathrm{HCN}(1-0)$ is from MTH08. b) The $\mathrm{HCN}(1-0) / \mathrm{HNC}(1-0)$ line ratio with the greyscale ranging from 0.5 to 2.0 and contours are in steps of 0.45 for the resolution of the $\mathrm{HNC}_{(1-0)}$ dataset. c) $\mathrm{The}^{\mathrm{HCO}}+(1-$ $0) / \mathrm{N}_{2} \mathrm{H}^{+}(1-0)$ line ratio with the greyscale ranging from 1.0 to 6.0 and contours are in steps of 1.0 . d) $\mathrm{The} \mathrm{HCO}^{+}(1-0) / \mathrm{HNC}^{-1-0)}$ line ratio with the greyscale ranging from 0.5 to 2.0 and contours are in steps of 0.25 .
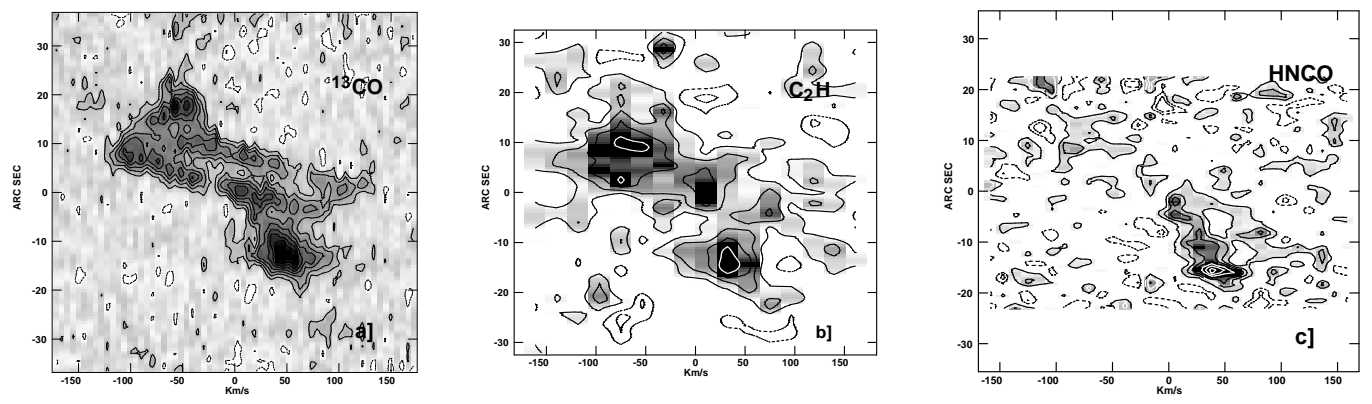

FIG. 8. - The Position-Velocity diagrams for a) ${ }^{13} \mathrm{CO}(1-0)$ (MTH08) $)$ b) $\mathrm{CCH}(1-0 ; 3 / 2-1 / 2)$, and $\left.c\right) \mathrm{HNCO}\left(5_{05}-4_{04}\right)$. The reference locations for each map are within $\pm 1^{\prime \prime}$ of $02^{h} 41^{m} 55^{s}, 59^{\circ} 36^{\prime} 10^{\prime \prime}$ and $\pm 2 \mathrm{~km} \mathrm{~s}^{-1}$ of $-20 \mathrm{~km} \mathrm{~s}^{-1}$. The position angle is taken at $29^{\circ}$. 


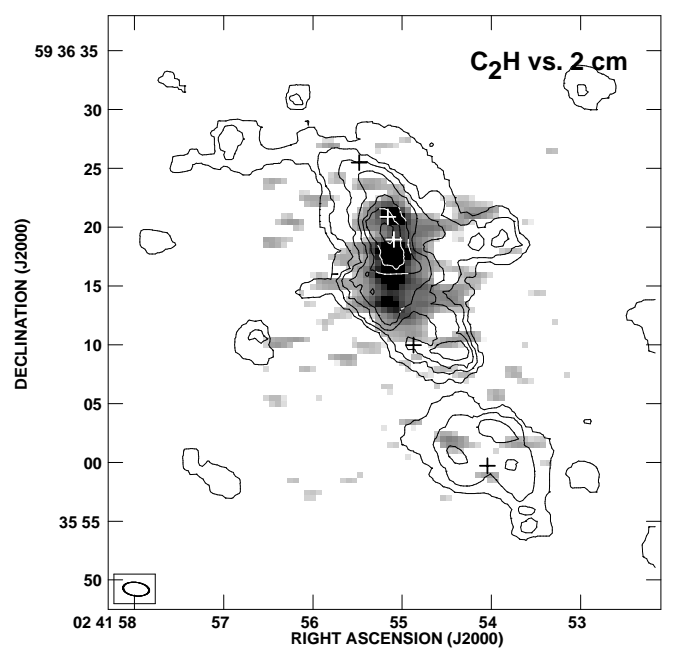

Fig. 9. $-\mathrm{C}_{2} \mathrm{H}$ contours overlaid on the $2 \mathrm{~cm}$ radio continuum (Turner \& Ho 1994). Contours are the same as in Fig. 3 and the greyscale ranges from $0.3 \mathrm{mJy} \mathrm{bm}^{-1}$ to $2 \mathrm{mJy} \mathrm{bm}^{-1}$ for a beam of $2 .^{\prime \prime} 2 \times 1$. $^{\prime \prime}$.

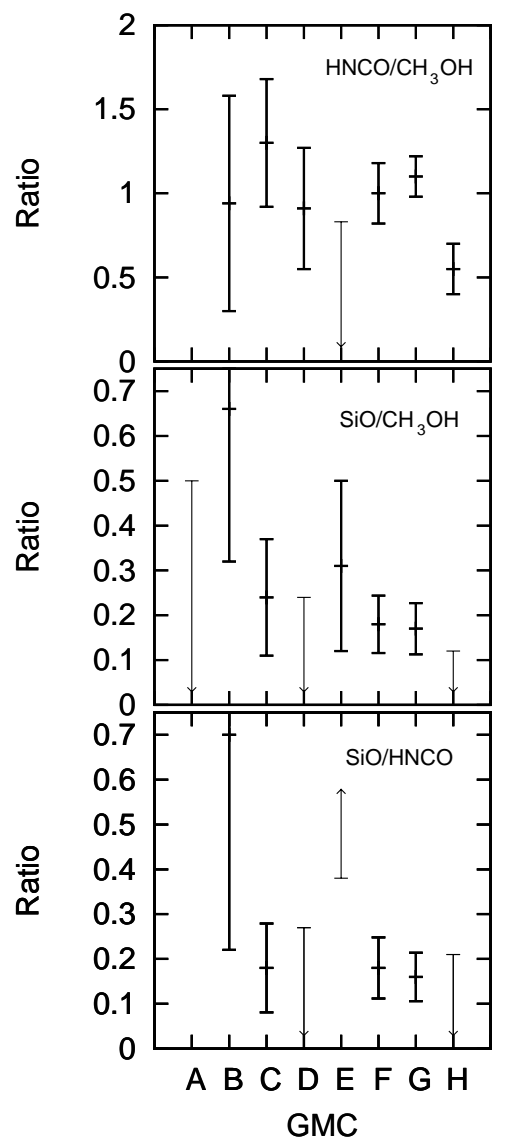

FIG. 10.- The shock tracer peak antenna temperature ratios. Ratios are measured over an $8^{\prime \prime}$ aperture centered on each cloud. Downward (upward) facing arrows are upper (lower) limits. 


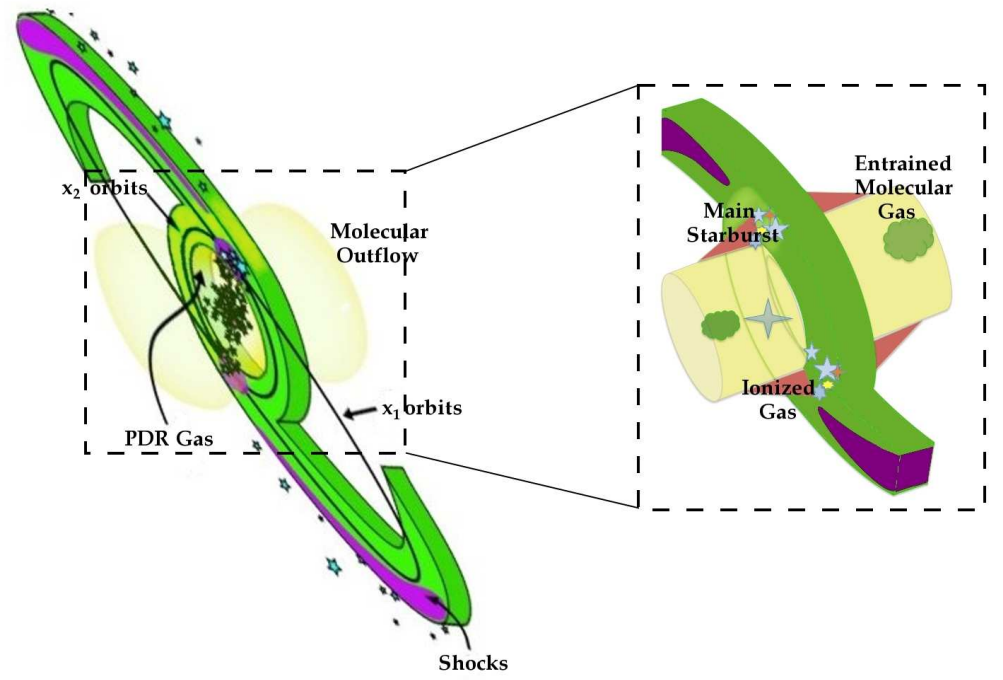

FIG. 11. - Schematic of the overall chemistry of Maffei 2. Left) The schematic adapted from Fig. 10 of MT05 to emphasize the similarity between the two nuclei's chemical properties. Right) A zoom in of the central molecular ring region with its star formation and molecular outflow.

TABLE 4

Molecular Abundances in Maffei 2

\begin{tabular}{lccccccccccc}
\hline \hline & $\mathrm{N}\left(\mathrm{H}_{2}\right)$ & $\mathrm{SiO}$ & $\mathrm{C}_{2} \mathrm{H}$ & $\mathrm{C}_{2} \mathrm{H}_{\text {cor }}{ }^{a}$ & $\mathrm{HCO}^{+}$ & $\mathrm{HNC}$ & $\mathrm{HC}_{3} \mathrm{~N}$ & $\mathrm{~N}_{2} \mathrm{H}^{+}$ & $\mathrm{C}^{34} \mathrm{~S}^{2} \mathrm{CH}_{3} \mathrm{OH}$ & $\mathrm{HNCO}^{2}$ \\
\hline $\mathrm{A}$ & $2.8(21)$ & $<6.8(-10)$ & $<5.4(-8)$ & $\ldots$ & $5.4(-9)$ & $<1.6(-9)$ & $<2.4(-9)$ & $<7.9(-10)$ & $<3.2(-9)$ & $<1.8(-8)$ & $\ldots$ \\
$\mathrm{B}$ & $9.7(21)$ & $<2.0(-10)$ & $<1.5(-8)$ & $\ldots$ & $6.1(-9)$ & $1.4(-9)$ & $<6.9(-10)$ & $6.1(-10)$ & $<9.2(-10)$ & $1.6(-8)$ & $<1.6(-8)$ \\
$\mathrm{C}$ & $1.9(22)$ & $<1.0(-10)$ & $1.8(-8)$ & $1.8(-8)$ & $4.2(-9)$ & $1.9(-9)$ & $1.0(-9)$ & $5.8(-10)$ & $<4.9(-10)$ & $4.2(-9)$ & $<2.4(-8)$ \\
$\mathrm{D}$ & $2.9(22)$ & $1.6(-10)$ & $2.2(-8)$ & $4.2(-8)$ & $6.0(-9)$ & $2.1(-9)$ & $7.6(-10)$ & $3.8(-10)$ & $<3.0(-11)$ & $3.0(-9)$ & $\sim 1.1(-9)$ \\
$\mathrm{E}$ & $3.0(22)$ & $\sim 4.7(-11)$ & $2.5(-8)$ & $4.8(-8)$ & $4.1(-9)$ & $2.2(-9)$ & $4.7(-10)$ & $2.6(-10)$ & $<3.0(-10)$ & $3.7(-9)$ & $<1.5(-9)$ \\
$\mathrm{F}$ & $2.3(22)$ & $\lesssim 8.3(-11)$ & $9.6(-9)$ & $9.6(-9)$ & $3.5(-9)$ & $2.3(-9)$ & $1.2(-9)$ & $7.4(-10)$ & $8.7(-10)$ & $1.0(-8)$ & $8.7(-9)$ \\
$\mathrm{G}$ & $2.0(22)$ & $\sim 1.1(-10)$ & $1.2(-8)$ & $2.3(-8)$ & $7.2(-10)$ & $1.3(-9)$ & $<5.0(-10)$ & $6.0(-10)$ & $\sim 6.5(-10)$ & $2.0(-8)$ & $1.6(-8)$ \\
$\mathrm{H}$ & $8.9(21)$ & $<2.1(-10)$ & $<1.7(8)$ & $<2.0(-9)$ & $<1.6(-9)$ & $1.3(-9)$ & $<7.5(-10)$ & $<2.5(-10)$ & $<1.0(-9)$ & $1.5(-8)$ & $<5.1(-9)$ \\
& & & & & & & & & &
\end{tabular}

Note. - Format for entries are $\mathrm{a}(\mathrm{b})=\mathrm{a} \times 10^{b}$. Each molecule is based on the assumptions of optically thin line emission with $\mathrm{T}_{e x}$ also 10 K. Upper limits are $2 \sigma$. Uncertainties are dominated by systematics and are at least a factor of 3 (see text for discussion of uncertainties). $\mathrm{H}_{2}$ column densities are based on ${ }^{13} \mathrm{CO}(1-0)$ emission sampled at the resolution of the $\mathrm{HNC}$ data with an abundance of $\left[\mathrm{H}_{2} /{ }^{13} \mathrm{CO}\right]=7.06 \times 10^{5}$ adopted (MTH08).

${ }^{a}$ Corrected for finite optical depth based of the fine structure line ratios (Table 1 .

TABLE 5

Other OBSERved Transitions

\begin{tabular}{|c|c|c|c|c|}
\hline Molecule & Transition & $\begin{array}{c}\nu \\
(G H z)\end{array}$ & $\begin{array}{c}\mathrm{T}_{m b} \\
(m J y / b m)\end{array}$ & GMCs \\
\hline $\mathrm{HCO}$ & $1_{0,1}-0_{0,0} ; \frac{1 / 2}{1 / 2} ; 1-1$ & 86.777 & $<20$ & $\cdots$ \\
\hline $\mathrm{HCO}$ & $1_{0,1}-0_{0,0} ; \frac{1 / 2}{1 / 2} ; 0-1$ & 86.806 & $<20$ & $\cdots$ \\
\hline $\mathrm{HN}^{13} \mathrm{C}$ & $1-0$ & 87.091 & $<22$ & $\cdots$ \\
\hline $\begin{array}{l}\mathrm{HCC}^{13} \mathrm{CN} / \mathrm{HC}^{13} \mathrm{C}_{2} \mathrm{~N} \\
\ldots\end{array}$ & $10-9$ & $90.593 / 90.602$ & $<22$ & $\cdots$ \\
\hline $\begin{array}{l}\cdots \\
\cdots\end{array}$ & $\begin{array}{l}\cdots \\
\cdots\end{array}$ & $\begin{array}{l}89.222(5) \\
90.912(5)\end{array}$ & $\begin{array}{c}70 \pm 15 \\
10 \pm 6\end{array}$ & $\begin{array}{l}\mathrm{C} \\
\mathrm{F}\end{array}$ \\
\hline
\end{tabular}

Note. - Upper limits are $2 \sigma$. 
TABLE 6

Selected Intensities Ratios

\begin{tabular}{|c|c|c|c|c|c|c|}
\hline Location & $\frac{H C N(1-0)}{H N C(1-0)}$ & $\frac{C C H(3 / 2-1 / 2)}{C C H(1 / 2-1 / 2)}$ & $\frac{H C N(1-0)}{H C O^{+}(1-0)}$ & $\frac{H N C(1-0)}{H N^{13} C(1-0)}$ & $\frac{C S(2-1)}{C^{34} S(2-1)}$ & $\frac{H C N(1-0)}{C S(2-1)}$ \\
\hline $\mathrm{A}$ & $\ldots$ & $\ldots$ & $\ldots$ & $>0.79$ & $>1.4$ & $\lesssim 1.6$ \\
\hline B & $2.4 \pm 0.4$ & $\cdots$ & $0.63 \pm 0.1$ & $>4.5$ & $>1.4$ & $4.9 \pm 1.5$ \\
\hline $\mathrm{C}$ & $1.6 \pm 0.2$ & $2.8 \pm 1.5$ & $0.90 \pm 0.1$ & $>6.6$ & $>6.0$ & $2.5 \pm 0.8$ \\
\hline D & $1.3 \pm 0.2$ & $1.5 \pm 0.3$ & $1.1 \pm 0.2$ & $>10$ & $>14$ & $2.1 \pm 0.6$ \\
\hline $\mathrm{E}$ & $1.3 \pm 0.2$ & $1.5 \pm 0.3$ & $1.3 \pm 0.2$ & $>9.4$ & $>13$ & $2.2 \pm 0.7$ \\
\hline $\mathrm{F}$ & $1.1 \pm 0.2$ & $2.8 \pm 1.8$ & $1.7 \pm 0.2$ & $>16$ & $\sim 4.1$ & $2.4 \pm 0.7$ \\
\hline $\mathrm{G}$ & $0.9 \pm 0.1$ & $1.5 \pm 0.7$ & $\sim 2.2$ & $>11$ & $\sim 3.8$ & $1.1 \pm 0.4$ \\
\hline $\mathrm{H}$ & $1.0 \pm 0.1$ & $\ldots$ & $\cdots$ & $>4.4$ & $>1.0$ & $<2.4$ \\
\hline
\end{tabular}

NoTE. - The resolution of the measurements are set by the lowest resolution input maps. The HCN(1-0) is from MTH08 and the CS(2-1) from (Kuno et al. 2008). In determining the $\mathrm{CS} / \mathrm{C}^{34} \mathrm{~S}$ ratio it is assumed that $\mathrm{CS}$ is spatially completely resolved by the Kuno et al. (2008) map. If this assumption is incorrect true ratios will be slightly lower than those quoted. Uncertainties reflect absolute flux calibration except for the $\mathrm{C}_{2} \mathrm{H}$ ratios which are based on signal-to-noise. Upper limits are $1 \sigma$.

TABLE 7

PCA CORRELATION MATRIX

\begin{tabular}{|c|c|c|c|c|c|c|c|c|c|c|c|c|c|}
\hline Maps & ${ }^{13} \mathrm{CO}$ & $3 \mathrm{MM}$ & $\mathrm{C}_{2} \mathrm{H}\left(\frac{3}{2}\right)$ & $\mathrm{HCN}$ & $\mathrm{HCO}^{+}$ & $\mathrm{HNC}$ & $\mathrm{CH}_{3} \mathrm{OH}$ & HNCO & $\mathrm{N}_{2} \mathrm{H}^{+}$ & $\mathrm{HC}_{3} \mathrm{~N}$ & $\mathrm{C}^{34} \mathrm{~S}$ & $\mathrm{SiO}$ & $\mathrm{C}_{2} \mathrm{H}\left(\frac{1}{2}\right)$ \\
\hline${ }^{13} \mathrm{CO}$ & 1.00 & & & & & & & & & & & & \\
\hline $3 \mathrm{MM}$ & 0.88 & 1.00 & & & & & & & & & & & \\
\hline $\mathrm{C}_{2} \mathrm{H}\left(\frac{3}{2}\right)$ & 0.85 & 0.77 & 1.00 & & & & & & & & & & \\
\hline $\mathrm{HCN}^{2}$ & 0.93 & 0.92 & 0.84 & 1.00 & & & & & & & & & \\
\hline $\mathrm{HCO}^{+}$ & 0.89 & 0.88 & 0.81 & 0.98 & 1.00 & & & & & & & & \\
\hline $\mathrm{HNC}$ & 0.95 & 0.87 & 0.84 & 0.97 & 0.94 & 1.00 & & & & & & & \\
\hline $\mathrm{CH}_{3} \mathrm{OH}$ & 0.68 & 0.47 & 0.49 & 0.43 & 0.34 & 0.51 & 1.00 & & & & & & \\
\hline $\mathrm{HNCO}$ & 0.52 & 0.27 & 0.32 & 0.21 & 0.12 & 0.33 & 0.86 & 1.00 & & & & & \\
\hline $\mathrm{N}_{2} \mathrm{H}^{+}$ & 0.86 & 0.71 & 0.67 & 0.77 & 0.72 & 0.82 & 0.71 & 0.58 & 1.00 & & & & \\
\hline $\mathrm{HC}_{3} \mathrm{~N}$ & 0.54 & 0.53 & 0.41 & 0.62 & 0.60 & 0.62 & 0.32 & 0.16 & 0.63 & 1.00 & & & \\
\hline $\mathrm{C}^{34} \mathrm{~S}$ & 0.20 & 0.24 & 0.17 & 0.18 & 0.20 & 0.19 & 0.14 & 0.095 & 0.20 & 0.15 & 1.00 & & \\
\hline $\mathrm{SiO}$ & 0.38 & 0.23 & 0.34 & 0.34 & 0.30 & 0.38 & 0.35 & 0.30 & 0.35 & 0.31 & -0.023 & 1.00 & \\
\hline $\mathrm{C}_{2} \mathrm{H}\left(\frac{1}{2}\right)$ & 0.64 & 0.60 & 0.70 & 0.66 & 0.63 & 0.63 & 0.27 & 0.18 & 0.45 & 0.24 & 0.008 & 0.32 & 1.00 \\
\hline
\end{tabular}

${ }^{\mathrm{a}}$ Data from (MTH08).

TABLE 8

PCA EIGENVECTORS

\begin{tabular}{|c|c|c|c|c|c|c|c|}
\hline PCA Comp. & 1 & 2 & 3 & 4 & 5 & 6 & 7 \\
\hline${ }^{13} \mathrm{CO}$ & 0.35 & -0.045 & -0.0043 & 0.11 & -0.042 & 0.15 & -0.064 \\
\hline $3 \mathrm{MM}$ & 0.32 & 0.15 & -0.10 & 0.12 & -0.086 & 0.19 & -0.50 \\
\hline $\mathrm{C}_{2} \mathrm{H}\left(\frac{3}{2}\right)$ & 0.31 & 0.097 & 0.086 & 0.24 & 0.12 & 0.020 & 0.81 \\
\hline $\mathrm{HCN}^{2}$ & 0.34 & 0.21 & 0.00 & -0.012 & -0.057 & 0.16 & -0.086 \\
\hline $\mathrm{HCO}^{+}$ & 0.33 & 0.28 & -0.029 & -0.024 & -0.055 & 0.21 & -0.038 \\
\hline $\mathrm{HNC}$ & 0.35 & 0.11 & 0.0015 & -0.031 & -0.054 & 0.16 & 0.038 \\
\hline $\mathrm{CH}_{3} \mathrm{OH}$ & 0.23 & -0.55 & -0.03 & 0.10 & -0.091 & 0.0061 & -0.031 \\
\hline HNCO & 0.17 & -0.65 & -0.01 & 0.17 & -0.050 & -0.10 & -0.061 \\
\hline $\mathrm{N}_{2} \mathrm{H}^{+}$ & 0.32 & -0.19 & -0.089 & -0.12 & -0.20 & -0.061 & 0.070 \\
\hline $\mathrm{HC}_{3} \mathrm{~N}$ & 0.23 & 0.067 & -0.13 & -0.68 & -0.29 & -0.52 & 0.086 \\
\hline $\mathrm{C}^{34} \mathrm{~S}$ & 0.080 & -0.0027 & -0.81 & 0.0092 & 0.56 & -0.11 & -0.0031 \\
\hline $\mathrm{SiO}$ & 0.16 & -0.19 & 0.43 & -0.51 & 0.66 & 0.23 & -0.074 \\
\hline $\mathrm{C}_{2} \mathrm{H}\left(\frac{1}{2}\right)$ & 0.24 & 0.19 & 0.33 & 0.37 & 0.29 & -0.70 & -0.23 \\
\hline Egnv. \% & 59 & 13 & 8.3 & 6.6 & 5.6 & 2.7 & 1.5 \\
\hline
\end{tabular}

